\title{
菱沸石分子篮膜的合成调控与气体分离研究进展
}

\author{
李子宜 ${ }^{1}$, 章佳佳 ${ }^{1}$, 邹小勤 ${ }^{2}$, 左家玉 ${ }^{1}$, 李 俊 $^{1}$, 刘应书 ${ }^{1}$, 裴有康 ${ }^{3,4}$ \\ (1. 北京科技大学 能源与环境工程学院, 北京 $100083 ; 2$. 东北师范大学 化学学院, 长春 $130024 ; 3$. 香港中文大 \\ 学(深圳) 理工学院, 深圳 518172; 4. 明尼苏达大学 机械工程系, 明尼阿波利斯 55455, 美国) \\ 摘 要: 菱沸石(Chabazite, $\mathrm{CHA})$ 分子笁膜因其八元环小孔 $(0.38 \mathrm{~nm})$ 三维孔道结构、可调的表面特性、较高的材料稳 \\ 定性与制备可重复性, 在轻质气体分离方面具有优异性能, 近年来逐渐成为分子笁膜研究热点之一。本综述介绍了 \\ 两种 CHA 分子篮膜(SAPO-34 膜、SSZ-13 膜)的基本特性，对比了 CHA 分子笁膜的合成方法(原位合成法、二次生 \\ 长法、微波加热法)优缺点及其应用现状，并重点针对主流的二次生长法制备 SSZ-13 膜与 SAPO-34 膜过程中关键 \\ 条件对薄膜质量的影响规律进行了详细阐述, 包括铺种条件(载体种类、晶种类别、铺种方式), 水热合成条件(晶化 \\ 时间、晶化温度、含水量、硅铝比、模板剂、阳离子种类)与煅烧方式(常规㷽烧、分段煅烧、快速热处理), 经细化 \\ 分析总结出上述两种膜的优选合成条件; 并进一步汇总了 $\mathrm{CHA}$ 分子篮膜表面化学调控(硅铝比调控、阳离子交换、 \\ 杂原子替换、氨基功能化、表面修饰)对气体分离增强的策略, 总结了 $\mathrm{CHA}$ 分子篮膜在各种气体体系中的分离特点 \\ 与单组分气体渗透特性。最后，对 CHA 分子篮膜今后的发展和应用前景进行了展望。
}

关＼cjkstart键＼cjkstart词：分子笁膜; 菱沸石; 合成制备; 表面化学调控; 气体分离; 综述

中图分类号: TQ127 文献标志码: A

\section{Synthesis and Gas Separation of Chabazite Zeolite Membranes}

\author{
LI Ziyi $^{1}$, ZHANG Jiajia ${ }^{1}$, ZOU Xiaoqin ${ }^{2}$, ZUO Jiayu ${ }^{1}$, LI Jun ${ }^{1}$, LIU Yingshu ${ }^{1}$, PUI David Youhong ${ }^{3,4}$
}

(1. School of Energy and Environmental Engineering, University of Science and Technology Beijing, Beijing 100083, China; 2. Institute of Chemistry, Northeast Normal University, Changchun 130024, China; 3. School of Science and Engineering, The Chinese University of Hong Kong, Shenzhen 518172, China; 4. Department of Engineering, University of Minnesota, Minneapolis 55455, USA)

\begin{abstract}
Chabazite (CHA) zeolite membranes exhibit superior performances in light gas separations owing to the eight-membered ring channel structure with small pore size $(0.38 \mathrm{~nm})$, adjustable surface characteristics, and high material stability and preparation reproducibility, and have gradually become one of the hot spots of zeolite membrane research in recent years. This review article first introduces the basic characteristics, the two typical CHA zeolite membranes (SAPO-34 and SSZ-13 membranes), then compares the synthesis and preparation methods of CHA zeolite membranes (in-situ synthesis, secondary growth synthesis, microwave heating methods) and analyzes their advantages and disadvantages in application status. The influences of their key synthesis conditions of the secondary growth as the
\end{abstract}

收稿日期: 2020-09-22; 收到修改稿日期：2020-10-27; 网络出版日期：2020-12-10

基金项目: 国家自然科学基金青年项目(21808012); 中央高校基本科研业务费(FRF-IDRY-19-025)

National Natural Science Foundation of China (21808012); Fundamental Research Funds for the Central Universities (FRF-IDRY-19-025)

作者简介: 李子宜(1990-), 男，副教授.E-mail: ziyili@ustb.edu.cn

LI Ziyi(1990-), male, associate professor. E-mail: ziyili@ustb.edu.cn

通信作者: 刘应书，教授. E-mail: ysliu@ustb.edu.cn

LIU Yingshu, professor. E-mail: ysliu@ustb.edu.cn 
mainstream synthesis method on the qualities of SSZ-13 and SAPO-34 membranes have been elaborated in detail, mainly including 1) the seeding conditions, such as carrier type, seeding crystal and seeding approach; 2) the hydrothermal synthesis conditions, such as crystallization time and temperature, water content, silica-to-alumina ratio, structure directing agent, and cation type; 3) the calcination approaches, such as conventional calcination, staged calcination, and rapid heating treatments. After comparative analysis, the preferred synthesis conditions of the above two typical CHA zeolite membranes are proposed. Furthermore, the modulation of membrane surface chemistry is discussed for the enhancement in gas separation, such as silica-to-alumina ratio adjustment, ion exchange, heteroatom substitution, amino-group functionalization, and surface modification. The detailed characteristics of gas separation in various gas mixture systems and the permeation properties of different single gases on CHA zeolite membranes are analyzed and summarized as well. Finally, the future development of CHA zeolite membranes is prospected.

Key words: membrane; chabazite; synthesis; surface chemistry modulation; gas separation; review

膜分离技术作为一种高效节能的环保分离技术， 在工业产品气制取、废气综合利用及环境保护等方 面具有广阔的应用前景。相对于传统的蒸馏、萃取、 重结晶等分离方法, 膜分离技术具有低能耗、低成 本、低污染、高效率等优势。沸石分子篮膜作为无 机多孔膜的重要成员之一，除了具备耐高温性、抗 微生物性、结构稳定性好、化学性能稳定等优点之 外, 还因与大多分子尺寸相近的孔道结构以及结构 中的较窄孔径分布和均一孔道, 具有在分子尺度上 对不同动力学直径分子的分离选择性、较强的设计 性与适应性 ${ }^{[1]}$ 。相比中孔(如: MFI)或大孔分子篎(如: FAU) 薄膜 ${ }^{[2-3]}$, 以 LTA、CHA、DDR、AEI 等为代 表的八元环小孔分子篮膜, 主孔径一般小于 $0.4 \mathrm{~nm}$, 在诸多轻质气体分离体系中(如: $\mathrm{H}_{2} 、 \mathrm{CO}_{2} 、 \mathrm{Xe}$ )备受 青睐 ${ }^{[4]}$ 。

近 20 年来, CHA 分子篮膜的发表文章数量呈逐 年上升趋势, 截止到目前已达到约 180 篇, 在几大 常见的八元环分子篮膜中呈现一定的优势(图 1)。 CHA 分子篮膜具有 $0.38 \mathrm{~nm} \times 0.38 \mathrm{~nm}$ 的孔径和 $17.3 \%$ 孔隙度, 对 $\mathrm{CO}_{2} /$ 烃类、 $\mathrm{H}_{2} /$ 其他小分子等混合 物具有较大的篮分作用, 对于 $\mathrm{CO}_{2} / \mathrm{CH}_{4}$ 分离, 分离 选择性最高可以达到 $270^{[5]}$; 此外, 还因高对称度、 开放的三维结构而具备较高的制备可重复性与工业 适用性 ${ }^{[6]}$ 。SSZ-13 膜和 SAPO-34 膜分别是硅铝、磷 铝酸盐的具有 CHA 拓扑结构的两种分子篮膜, 前 者最早由 Falconer 等 ${ }^{[7]}$ 通过二次生长法成功合成, 具有硅铝比可调、耐酸、水热稳定性好等优点，同 时也存在合成周期较长、合成影响因素较多、模板 剂昂贵等缺点; 后者最早由 Zhang 等 ${ }^{[8]}$ 通过原位合 成法合成得到, 具有热稳定性好、水热性能稳定等 优点, 相较于 SSZ-13 膜更易吸附极性分子, 但仅具 有中等的耐酸性, 常温下易吸水, 同时具有硅铝比

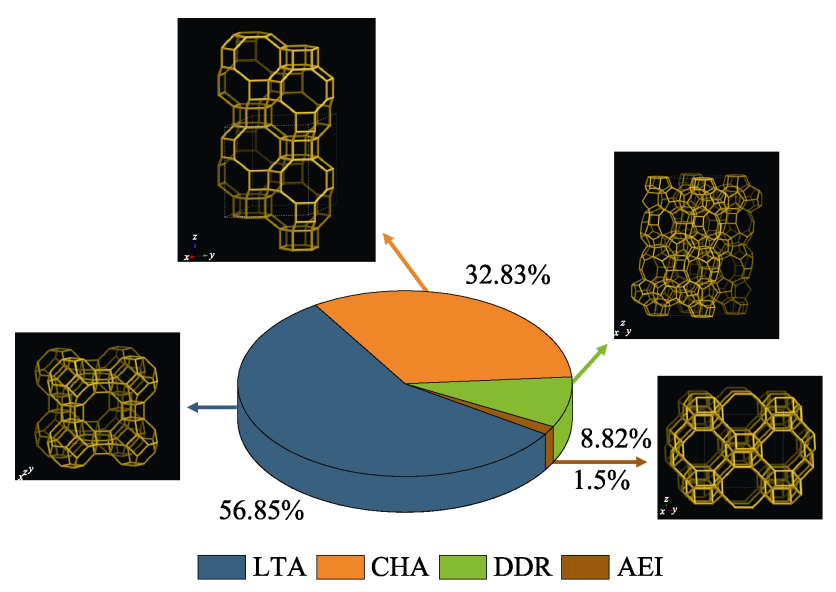

图 1 八元环分子篮膜相关出版文章比例

Fig. 1 Proportions of publications and structures for primary 8 -membered ring zeolite members

调节范围较小、合成影响因素较多等不足 ${ }^{[9]}$ 。

分子篮膜的气体分离一般分为表面吸附和孔道 扩散两个过程 ${ }^{[1,10]}$ 。首先, 不同的分子吸附在分子篮 膜表面; 其次, 在化学势的作用下, 分子从一个吸 附位点跃迁至下一个吸附位点或空位 ${ }^{[11]}$, 从而进入 分子孔道并扩散至渗透端。分子篮膜的表面特性与 气体分子极性决定了表面吸附强度, 孔径尺寸及构 型与气体分子动力学直径的关系决定了孔道扩散特 性。CHA 分子篎膜具有与诸多轻质气体动力学直径 相近的孔径, 同时可通过改变硅铝比调控表面的亲 疏水性 ${ }^{[12]}$, 易于形成较为显著的膜内扩散差异(极 端情况下为分子篮分效应 $)^{[13]}$, 是目前分离轻质气 体被认可的理想分子篮膜材料之一 ${ }^{[14]}$ 。本文重点综 述当前国内外 CHA 分子篎膜的合成方法与气体分 离研究现状, 阐释合成条件对薄膜质量以及薄膜结 构对气体分离性能的影响规律, 并对其未来发展和 应用前景作出了展望。 


\section{CHA 分子篮膜的合成方法}

\section{1 原位合成法}

原位合成法是将载体直接放置于合成溶液中, 使分子篮直接在载体表面成核、生长, 形成分子篮 膜的传统方法(图 2)。该法操作简单且所需合成设备 简易, 但难以一次性合成连续且致密的薄膜, 通常 需采用多次原位合成弥补缺陷，但这样又会增加合成 复杂性并可能导致已合成晶体转晶或溶解 ${ }^{[5-16]}$ 。另外, 原位合成法无法保证分子篮晶体优先生长于载体表 面，不易控制膜厚，易导致膜过厚或不均匀 ${ }^{[17]}$ 。当前 采用原位合成法制备 $\mathrm{CHA}$ 分子篮膜的成功案例较 少, 主要探索集中在 SAPO-34 膜的制备上, 如 $\mathrm{Hong}^{[18]} 、 \mathrm{Li}^{[19]}$ 等利用原位合成法制备出 SAPO-34 膜, 分别用于氢气纯化与 $\mathrm{CO}_{2} / \mathrm{CH}_{4}$ 分离。

\section{2 二次生长法}

二次生长法是预先在载体表面涂敷晶种并作为 生长中心继续生长成膜的一种合成方法 ${ }^{[20]}$ (图 2), 该方法可缩短合成时间，提高合成效率，而且能更 好地控制膜层厚度和晶体微观结构。此外, 晶种在 载体表面的修饰作用还可减少载体对成膜过程的影 响, 避免缺陷的产生。目前国内外从事 $\mathrm{CHA}$ 分子篎 膜合成研究的主要团队, 基本采用二次生长法, 其 中包括最初合成 CHA 分子篎膜的科罗拉多大学的 Falconer 和 Noble 等 ${ }^{[5,21-23]}$ 。针对 SSZ-13 膜
的二次生长法合成，国外课题组例如 Kosinov 和 Choi 等 ${ }^{[14,24-26]}$ 成功合成出薄而均匀且分离性能良好 的高硅 SSZ-13 膜; 国内的顾学红、周荣飞、陈祥树 等 ${ }^{[27-34]}$ 得到了渗透性较高以及分离性能良好的 SSZ-13 膜。针对 SAPO-34 膜的二次生长法, 国外的 Carreon、Yu 和 Funke 等 ${ }^{[35-40]}$ 有着多年的研究经验，成 功制备出对轻质气体具有良好分离效果的 SAPO-34 膜, 国内的顾学红、张延风等 ${ }^{\left[{ }^{[1-44]}\right.}$ 也成功合成出渗 透性能以及分离性能优良的致密 SAPO-34 膜, 用于 $\mathrm{CO}_{2} / \mathrm{CH}_{4}$ 和甲醇/碳酸二甲酯分离。在二次生长法合 成 CHA 分子篎膜过程中, 不同的铺种、水热合成与 炦烧条件都会对膜的结构、致密度、气体渗透性及 分离选择性产生不同程度的影响。针对实际工业应 用所需的 CHA 分子篣膜大面积、规模化批量生产, 二次生长法亦是首选方法, Sato 等 ${ }^{[55]}$ 率先在面积为 $45 \mathrm{~cm}^{2}$ 的管状载体上合成了具有高重现性的高硅 SSZ-13 膜; $\mathrm{Li}$ 等 ${ }^{[1]}$ 在面积为 $39 \mathrm{~cm}^{2}$ 的载体上成功合 成了高质量的管状 SAPO-34 膜。

\section{3 微波加热法}

微波加热法是微波辐射下的水热合成法，通过 离子振荡和偶极子旋转使正常的水分子氢键发生旋 转, 分离出的活性水分子具有更高的溶解凝胶的能 力(图 2)。与常规加热相比，合成的晶体尺寸小而均 匀, 缩短了合成时间，提高合成效率 ${ }^{[46]}$ 。基于微波 加热法, $\mathrm{Hu}$ 等 ${ }^{[47]}$ 制备出薄(厚度为 $4 \mu \mathrm{m}$ )且致密的
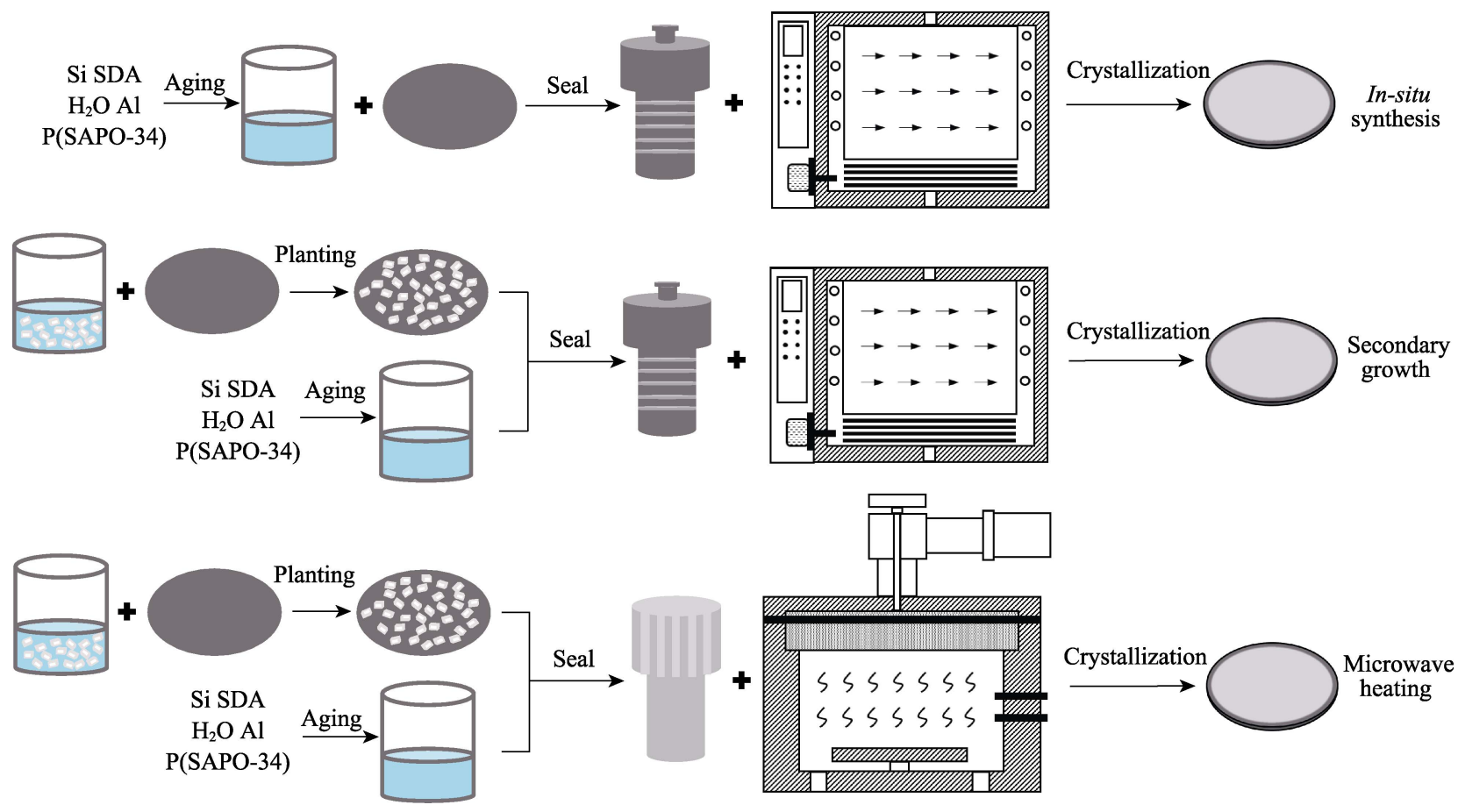

图 2 CHA 分子篮膜制备方法流程示意图

Fig. 2 Schematic diagrams of CHA zeolite membrane preparation methods 
表 1 CHA 分子篮膜合成方法对比

Table 1 Comparison of CHA zeolite membrane synthesis methods

\begin{tabular}{|c|c|c|c|}
\hline Method & Advantage & Disadvantage & Status of use \\
\hline In-situ synthesis & $\begin{array}{l}\text { (1) Simple production equipment } \\
\text { (2) Easy to use }\end{array}$ & $\begin{array}{l}\text { (1) Low success rate } \\
\text { (2) Long synthesis time } \\
\text { (3) Difficult to control }\end{array}$ & $\begin{array}{l}\text { Less research, basically used for the } \\
\text { synthesis of SAPO- } 34 \text { membrane }\end{array}$ \\
\hline Secondary growth & $\begin{array}{l}\text { (1) Simple production equipment } \\
\text { (2) High success rate } \\
\text { (3) Short synthesis time }\end{array}$ & (1) Tedious steps & $\begin{array}{l}\text { More research, conducive to large-scale } \\
\text { mass production }\end{array}$ \\
\hline Microwave heating & $\begin{array}{l}\text { (1) High success rate } \\
\text { (2) Short synthesis time }\end{array}$ & $\begin{array}{l}\text { (1) Tedious steps } \\
\text { (2) High equipment cost } \\
\text { (3) High energy consumption }\end{array}$ & $\begin{array}{l}\text { New method, still in basic research } \\
\text { stage }\end{array}$ \\
\hline
\end{tabular}

SSZ-13 膜, 用于乙醇和异丙醇的水溶液分离, 较常 规加热, 合成时间缩短为原来的 $1 / 3$, 渗透通量提升 了一倍; Liu 等 ${ }^{[48]}$ 合成出尺寸均匀的 SAPO-34 晶体 $(400 \mathrm{~nm})$ 与薄 $(3 \mu \mathrm{m})$ 且致密的 SAPO-34 膜; 较常规 加热, 合成时间缩短为原来的 $1 / 4$; Chew 等 ${ }^{[49]}$ 在短 时间内 $(2 \mathrm{~h})$ 成功合成出尺寸均匀 $(<1 \mu \mathrm{m})$ 的 SAPO-34 晶体与较薄 $(3 \sim 4 \mu \mathrm{m})$ 的 SAPO-34 膜, 用于 $\mathrm{CO}_{2} / \mathrm{CH}_{4}$ 分离。然而, 微波加热法所需的微波合成 设备成本与运行能耗较高, 距离实际产业化应用 尚远。

\section{4 合成方法对比}

上述几种 $\mathrm{CHA}$ 分子篎膜制备方法的优缺点及 使用现状见表 1 。针对实验条件、合成效率以及成 本等几方面综合考虑, 当前大多数研究者们仍然采 用二次生长法制备 CHA 分子篮膜, 并不断优化其 合成条件, 相关研究汇总详见本文第二节。

\section{2 二次生长法制备 CHA 分子篮膜的 影响因素}

\section{1 铺种条件的影响}

1)载体。在载体材料的选择上, $\alpha$-氧化铝和莫来 石的热膨胀系数与其他载体相比, 与 $\mathrm{CHA}$ 的相差 较小 ${ }^{[50-51]}$, 且其作为陶瓷材料与分子篮性质相似, 更易于膜层生长 ${ }^{[52]}$ 。针对 SSZ-13 膜, 周荣飞等 ${ }^{[27,31]}$ 基于莫来石管合成了致密的 SSZ-13 膜, Kosinov 和 Lee 等 ${ }^{[14,24-25]}$ 基于管状、片状 $\alpha$-氧化铝合成了性能 良好的 SSZ-13 膜。针对 SAPO-34 膜, 载体主要集 中在 $\alpha$-氧化铝上, 如 Chen 和 Liu 等 ${ }^{[41,53-55]}$ 均基于管 状 $\alpha$-氧化铝合成出高渗透通量且致密的 SAPO-34 膜。基于上述研究, 可以看出分子笁和载体具有相 似的化学组成, 对增强载体与分子篮之间的亲和力
起到促进作用: Kosinov 等 ${ }^{[56]}$ 在表面粗粘的对称氧 化铝上制备的 SSZ-13 膜在去除模板剂的同时能够 不产生裂纹; $\mathrm{Yu}$ 等 ${ }^{[57]}$ 同样通过载体表面改性, 在避 免合成混合物进入载体孔洞的同时允许沸石晶体在 载体表面上沉积, 从而得到高质量膜。

2)晶种。晶种是影响分子篮膜合成的关键因素, 晶体尺寸、铺种方式与晶种固定措施都会直接影响 最终膜的质量。

晶种是影响分子篮膜二次生长的关键因素, 晶 种尺寸越小, 其生长能力越强, 更易在载体表面附 着生长。纳米晶种可促进致密 $\mathrm{CHA}$ 分子篎膜的合

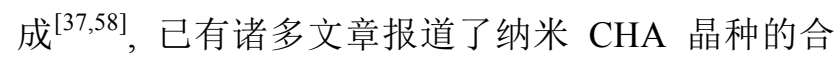
成方法 ${ }^{[59-63]}$, 亦有通过球磨方式将常见微米级 CHA 晶体研磨至纳米尺寸作为晶种, 虽所得晶种形状不 定, 但因仍保有 $\mathrm{CHA}$ 晶型而能够起到诱导合成的

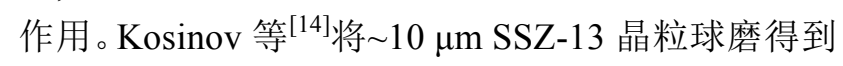
$120 \mathrm{~nm}$ 的晶种, 制备出分离性能良好的致密 SSZ-13 膜; Huang 等 ${ }^{[40]}$ 还发现使用大长径比的片状 纳米晶种可得到薄且连续的高质量 SAPO-34 膜。

铺种方式对合成平整 CHA 分子篎膜也起着关 键作用, 晶种在载体表面附着越均匀, 越有利于形 成高质量 CHA 分子篮膜。针对 SSZ-13 晶种, Karakilic 等 ${ }^{[64]}$ 发现采用浸涂法能获取更加均匀的晶 种层, 而旋涂和擦涂两种方法均难以得到均匀分布 的晶种层。针对 SAPO-34 晶种, Jabbari 等 ${ }^{[65]}$ 对擦涂、 浸涂、电泳沉积等铺种方法进行了比较, 发现通过 擦涂和电泳沉积能够产生更加均匀且致密的 SAPO-34 晶种层。

铺种后做进一步固定处理可避免因晶种在载体 上附着力较弱而落入后续合成液中, 部分工作者采 用加热方式固定晶种, 有时这会成为分子篎膜合成 的关键步骤。例如, Hong 等 ${ }^{[26]}$ 将球磨晶种附着于载 体表面后, 在 $450{ }^{\circ} \mathrm{C}$ 下煅烧用于固定晶种, 合成了 
致密平整的 SSZ-13 膜, 其表面沸石晶体结晶度良 好且呈立方型; Qiu 等 ${ }^{[66]}$ 采用球磨晶种铺于载体表 面，在 $200{ }^{\circ} \mathrm{C}$ 下煅烧用于固定晶种，合成的 SSZ-13 膜表面光滑平整，共生性良好。

\section{2 合成条件的影响}

1)晶化温度。晶化温度会对 CHA 晶体的成核速 率和生长速率均产生影响, 在允许范围内, 温度越 高, 成核和生长速率越快, 结晶度越好, 形成的晶 粒越大，但若是温度过高，晶体可能会发生结构的 转变, 产生杂相 ${ }^{[67]}$ (图 3)。Bohström 等 ${ }^{[68]}$ 探索了晶 化温度对 SSZ-13 晶种生长的影响, 发现 120 $130{ }^{\circ} \mathrm{C}$ 没有发生实质性的粒径生长, 140 150 ${ }^{\circ} \mathrm{C}$ 发生 缓慢粒径生长, $160{ }^{\circ} \mathrm{C}$ 下粒径快速生长, 在 170 $180{ }^{\circ} \mathrm{C}$ 趋于稳定, 超过 $180{ }^{\circ} \mathrm{C}$ 则会出现杂相, 得不 到纯相 SSZ-13。SSZ-13 膜与上述 SSZ-13 晶种的 合成温度范围基本相一致, 都在 $150 \sim 170{ }^{\circ} \mathrm{C}$ 范围 内 $^{[14,24,26-27]}$ 。相比之下, SAPO-34 膜与 SAPO-34 晶 种的合成温度存在一定差异, 前者主要在 180 $230{ }^{\circ} \mathrm{C}^{[41,53,66]}$, 后者基本在 $170 \sim 190{ }^{\circ} \mathrm{C}, 160{ }^{\circ} \mathrm{C}$ 下晶 化则会生成 AEI 沸石杂相 ${ }^{[69]}$ 。

2)晶化时间。晶化时间与 CHA 分子篮膜层的结 晶度与厚度密切相关, 通常要与晶化温度配合选取, 晶化时间过短会导致膜层不完整，晶化时间过长会 使厚度过大(图 3)。针对 SSZ-13 膜合成, 150 170 ${ }^{\circ} \mathrm{C}$ 下晶化时间大多控制在 $24 \sim 72 \mathrm{~h}^{[14,24]}$ 。Zheng 等 ${ }^{[27]}$ 在 $170{ }^{\circ} \mathrm{C}$ 下对 SSZ-13 膜的合成研究发现随着晶化 时间(8 48 h) 延长, 其结晶度和膜厚也随之增大, 晶 化 $48 \mathrm{~h}$ 后合成的膜中晶体交联共生性良好, 膜厚约 为 $10 \mu \mathrm{m}$, 其气体渗透行为与分离性能明显优于其 他晶化时间对应的结果。

SAPO-34 膜晶化时间较 SSZ-13 短, 通常不超过 $30 \mathrm{~h}$ 。Chen 等 ${ }^{[41]}$ 在 $180{ }^{\circ} \mathrm{C}$ 下对 SAPO-34 膜的合成 研究发现, 随着晶化时间(6 30 h)的延长, 表面晶粒 增大, 晶体共生行为更加明显, $18 \mathrm{~h}$ 时即可得到良好 的共生性; $\mathrm{Liu}$ 等 ${ }^{[48]}$ 在 $220{ }^{\circ} \mathrm{C}$ 晶化温度下探究了 $2 \sim 8 \mathrm{~h}$ 晶化时间内 SAPO-34 膜的生长规律, 发现晶化时间为 $6 \mathrm{~h}$ 时可形成连续且致密的膜, 共生性最佳。

3)含水量。针对 SSZ-13 膜, 含水量会对合成液 碱度产生影响, 合成液碱度过高会溶解部分晶体, 过 低会降低合成液原料的溶解度, Song 和 Liang 等 ${ }^{[29,32]}$ 基于 $1 \mathrm{SiO}_{2}: 0.05 \mathrm{Al}_{2} \mathrm{O}_{3}: 0.05 \mathrm{Na}_{2} \mathrm{O}: 0.5 \mathrm{TMAdaOH}$ (模板剂): $x \mathrm{H}_{2} \mathrm{O}(x=40 \sim 120)$ 的配方, 发现 $x=40 \sim 120$ 含水量的合成液都能够合成连续的 SSZ-13 膜, 随 着 $x$ 逐渐增加, 晶体形态从立方晶体变为球形晶体, $x=80$ 时的膜共生性与气体渗透性明显优于其他含
水量合成的结果。纯硅的 SSZ-13 膜是在含氟体系下 由固体前驱体和少量水的凝胶所制备，对合成液的 含水量要求较高, 当 $\mathrm{H}_{2} \mathrm{O} / \mathrm{SiO}_{2}>6$ 时易形成 STT-型 沸石 ${ }^{[70]}$ 。Kida 等 ${ }^{[71]}$ 采用 $1.0 \mathrm{SiO}_{2}: 0.8 \mathrm{TMAdaOH}$ : $0.8 \mathrm{HF}: 5.7 \mathrm{H}_{2} \mathrm{O}$ 的配方成功合成出连续致密的纯硅 SSZ-13 膜。

针对 SAPO-34 膜, 合成液含水量可直接影响 SAPO-34 膜的膜厚度, 探究含水量对 SAPO-34 膜生 长的影响规律, 对调控其膜厚具有重要意义。Carreon 等 ${ }^{[72-73]}$ 基于 $1.0 \mathrm{Al}_{2} \mathrm{O}_{3}: 1.0 \mathrm{P}_{2} \mathrm{O}_{5}: 0.3 \mathrm{SiO}_{2}: 1.0 \mathrm{TEAOH}$ (模板剂) : 1.6DPA(模板剂) : $x \mathrm{H}_{2} \mathrm{O}$ 的配方, 发现含 水量 $x$ 在 150 400 范围内均可得到连续致密 SAPO-34 膜, 提高含水量可降低膜厚, 实现膜厚在 $8.7 \sim 2.7 \mu \mathrm{m}$ 范围内的精确调控。

4)硅铝比。针对硅铝酸盐的 SSZ-13 膜, 硅铝比 是影响膜表面极性与膜生长特性的重要因素, 较低 的 $\mathrm{Al}$ 含量原则上可以产生更少的缺陷(图 3)。 Kosinov 等 ${ }^{[14,24]}$ 采用摩尔比为 $x \mathrm{SiO}_{2}: y \mathrm{Al}(\mathrm{OH})_{3}$ : $2 \mathrm{NaOH}: 2 \mathrm{TMAdaOH}: 440 \mathrm{H}_{2} \mathrm{O}(x+y=10.5, x / y=5 \sim 125)$ 的合成液, 发现随着硅铝比提高, SSZ-13 膜共生性 更好, 且高硅 SSZ-13 膜的气体渗透性和选择性更 稳定, 但硅铝比为 125 时会形成 AFI 型沸石杂相。

针对硅铝磷酸盐的 SAPO-34 膜，可调节的硅铝 比范围很有限, 通常在 0.15 附近, 也有研究人员探 索了其他比例， $\mathrm{Li}$ 等 ${ }^{[74]}$ 基于 $1.0 \mathrm{Al}_{2} \mathrm{O}_{3}: 1.0 \mathrm{P}_{2} \mathrm{O}_{5}$ : $x \mathrm{SiO}_{2}: 1.2 \mathrm{TEAOH}: 55 \mathrm{H}_{2} \mathrm{O}(x=0.1 \sim 0.6)$ 的配方, 发现 当 $x \geqslant 0.3$ 时才能合成出纯相 SAPO-34 膜, 且硅铝比 不会明显影响 SAPO-34 膜的孔道特性。

5)模板剂。CHA 分子篮膜可基于一种或多种模 板剂来制备。针对 SSZ-13 膜的合成，常采用昂贵的 $\mathrm{TMAdaOH}$ 作为模板剂, 因此研究人员希望找到廉 价模板剂作为代替品, 或形成混合模板剂以减少其 用量, 其中 $\mathrm{TEAOH}$ 是较适合的替代者, Zheng 等 ${ }^{[27]}$ 对比了双模板剂 (TEAOH、TMAdaOH)与单模板剂 (TMAdaOH) 所合成 SSZ-13 膜质量, 发现前者晶体 呈近立方晶形, 具有更高的结晶度, 且 $\mathrm{CO}_{2} / \mathrm{CH}_{4}$ 分离 系数远高于后者。针对 SAPO-34 膜的合成, 探究模 板剂的选取主要是为了提高膜合成效率，当前不同 模板剂对 SAPO-34 膜合成的影响尚不清晰, Kim 等 ${ }^{[75]}$ 发现使用传统单模板 TEAOH 较双模板剂(TEAOH、 DPA)合成的 SAPO-34 膜更薄 $(3 \sim 4 \mu \mathrm{m})$, 且具有更高 的气体渗透性和选择性; 而 Carreon 等 ${ }^{[23]}$ 却发现使用 双模板剂(TEAOH、DPA)合成的膜片质量更高，更 有利于气体分离。

6)阳离子种类。调控阳离子种类通常会改变 
CHA 分子篮膜表面的亲疏水性及催化特性, 也能起 到微调孔径的目的, 从而改善膜分离性能 ${ }^{[76]}$ 。此外, 通过改变阳离子种类亦可调控 $\mathrm{CHA}$ 分子篮膜的取 向生长, 日本东京大学的 Sato 等 ${ }^{[45]}$ 采用 $1 \mathrm{SiO}_{2}: 0.05$ $0.1 \mathrm{Al}_{2} \mathrm{O}_{3}:(0.05 \sim 0.1) \mathrm{Na}_{2} \mathrm{O}:(0.03 \sim 0.06) \mathrm{K}_{2} \mathrm{O}:(0.03 \sim 0.05)$ TMAdaOH: $(80 \sim 120) \mathrm{H}_{2} \mathrm{O}$ 的配方, 通过在合成液中 添加 $\mathrm{K}^{+}$得到一种能够在膜生长过程中选择性对 $[1,1,1]_{r}$ 轴方向优先结晶的高硅 SSZ-13 膜, 膜表面 形成了紧密排布的晶粒, 而晶体的 $[1,1,1]_{r}$ 轴方向垂 直于膜表面(图 3)。

\section{3 煅烧条件的影响}

完成水热合成后的 CHA 分子篮膜, 需脱除孔

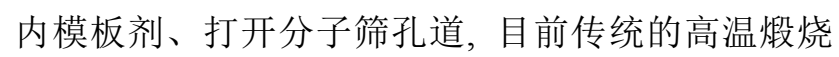
方式, 即将空气或者纯氧以一定流量通入盛放膜片 的马弗炉或者管式炉, 采用 $0.2 \sim 2{ }^{\circ} \mathrm{C} / \mathrm{min}$ 低升/降温 速率, 使膜在 400 550 ${ }^{\circ} \mathrm{C}$ 下煆烧 4 10 h, 模板剂被 氧化分解成气体, 从孔中逸出 ${ }^{[21-24,37-41,77-78]}$ 。由于分 子篮膜层与载体的热膨胀系数差异以及 $\mathrm{CHA}$ 分子 篮相较其他类型分子篮具有更高的孔隙率 $(17.3 \%)$, 高温㷽烧过程中大量气体的逸出容易导致分子篮膜 开裂, 产生晶界缺陷 ${ }^{[79]}$ 。
为解决上述问题, 有研究者提出并探索了分段 煅烧 ${ }^{[64]}$ 、快速热处理(Rapid Thermal Processing,

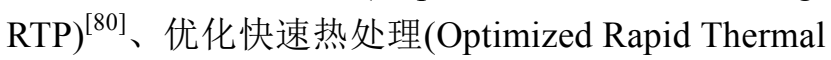

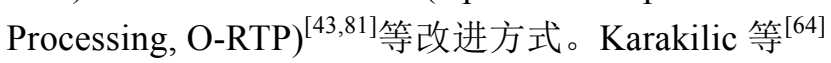
基于分段殷烧, 在升温过程中分别在 400 和 $450{ }^{\circ} \mathrm{C}$ 停留 $2 \mathrm{~h}$, 在 $500{ }^{\circ} \mathrm{C}$ 下保持 $2 \mathrm{~h}$ 后降温, 缩短分子篎 膜在高温下暴露时间的同时延长其在中间温度的煅 烧时间, 所得 SSZ-13 膜的气体渗透性能优于传统 煅烧得到的膜。Kim 等 ${ }^{[80]}$ 将快速热处理与常规煅烧 相结合, 将 SSZ-13 膜暴露于 $1000{ }^{\circ} \mathrm{C}$ 高温下 $1 \mathrm{~min}$ 后自然冷却, 再采用常规瑖烧方式, 快速升温可在 模板剂去除引起平面内拉应力积累之前, 使相邻晶 粒间的 $\mathrm{Si}-\mathrm{OH}$ 基团发生缩合, 增强晶粒间的粘结, 与常规炦烧所制得的 SSZ-13 膜相比更能抑制缺陷 的产生, $\mathrm{CO}_{2} / \mathrm{N}_{2}$ 和 $\mathrm{CO}_{2} / \mathrm{CH}_{4}$ 混合物的选择性均有显 著提高。Chang 和 Tang 等 ${ }^{[43,81]}$ 在上述方法基础上又 进行了优化, 即 $\mathrm{CHA}$ 分子篮膜暴露于 $700{ }^{\circ} \mathrm{C}$ 高温下 $1 \mathrm{~min}$ 后, 省略自然冷却以及升温过程, 直接进入预 热好的反应炉中进行高温炦烧以及低速率降温, 这 样的方式进一步加强了沸石晶体之间的键合, 缩短 了热暴露时间, $\mathrm{CO}_{2} / \mathrm{CH}_{4}$ 选择性提高了 2 倍。除了

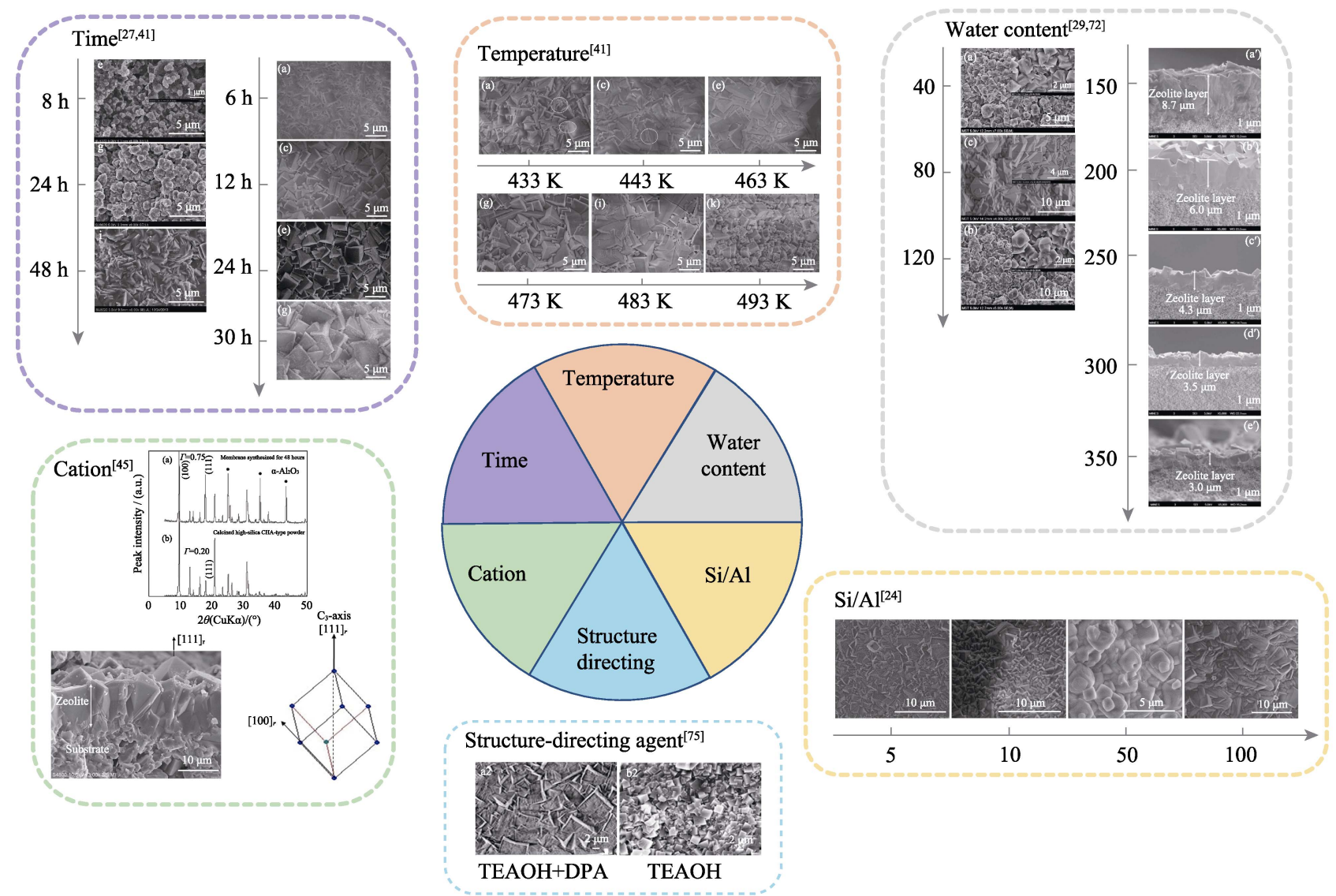

图 $3 \mathrm{CHA}$ 分子篮膜合成条件影响规律汇总图

Fig. 3 Summary of influences of CHA zeolite membrane synthesis conditions 
利用高温的手段外, Yang 等 ${ }^{[82]}$ 利用紫外线辐射的方 式对模板剂进行分解和去除，紫外线照射促进环境 氧的离解, 产生臭氧和原子氧, 同时也刺激分解了 模板剂，从而产生活性物质，如离子、自由基和激发 态分子等，这些活性有机物易受到原子氧或臭氧的 攻击, 转化为 $\mathrm{CO}_{2} 、 \mathrm{H}_{2} \mathrm{O}$ 和 $\mathrm{N}_{2}$ 逸出, 该方法可以在 近环境条件下进行，规避了高温衫烧时热膨胀所带 来的缺陷, $\mathrm{H}_{2} / \mathrm{C}_{3} \mathrm{H}_{8}$ 选择性提升了近 200 倍。

表 2 对 $\mathrm{CHA}$ 分子篮膜二次生长法的关键影响因 素进行了分析总结, 汇总得到 SSZ-13 和 SAPO-34 两 种典型 CHA 分子篮膜的优选合成条件。

\section{CHA 分子篮膜表面化学调控及气 体分离研究}

\subsection{CHA 分子耖膜表面化学调控策略}

1)硅铝比调控。通过调控 CHA 分子笁膜硅铝比 来促进气体分离主要体现在增强表面吸附与膜内扩 散两个方面。在增强表面吸附方面, 分子篮膜硅铝 比越低, 电荷补偿阳离子就越多, 越有利于偶极矩 或四极矩较大的气体分子吸附, 可促进如 $\mathrm{CO}_{2} / \mathrm{CH}_{4}$ 等 极性相差较大的混合体系的分离。Kosinov 等 ${ }^{[14]}$ 采 用硅铝比为 100 的 SSZ-13 膜获取了 $\mathrm{CO}_{2} / \mathrm{CH}_{4}$ 分离 系数 $43 、 \mathrm{CO}_{2}$ 渗透通量 $2.5 \times 10^{-7} \mathrm{~mol} \cdot \mathrm{m}^{-2} \cdot \mathrm{s}^{-1} \cdot \mathrm{Pa}^{-1} ; \mathrm{Wu}$ 等 ${ }^{[21]}$ 采用硅铝比为 20 的 SSZ-13 膜则在相近条件下 将 $\mathrm{CO}_{2} / \mathrm{CH}_{4}$ 分离系数提升至 $178, \mathrm{CO}_{2}$ 渗透通量仅仅 降低至 $2.1 \times 10^{-7} \mathrm{~mol} \cdot \mathrm{m}^{-2} \cdot \mathrm{s}^{-1} \cdot \mathrm{Pa}^{-1}$ 。

在增强膜内扩散方面, 硅铝比越高, 阳离子越
少, 膜内扩散位阻减小, 可促进如 $\mathrm{N}_{2} / \mathrm{CH}_{4}$ 等动力学 直径相差较大的混合体系的分离 ${ }^{[24,83]}$ (图 4(a))。Wu 和 $\mathrm{Li}$ 等 ${ }^{[21,31]}$ 分别合成了 $\mathrm{Si} / \mathrm{Al}=20 、 100$ 的 $\mathrm{SSZ}-13$ 膜用于 $\mathrm{N}_{2} / \mathrm{CH}_{4}$ 分离, 其 $\mathrm{N}_{2}$ 渗透率分别达到 $0.18 \times 10^{-7} 、 1.07 \times 10^{-7} \mathrm{~mol} \cdot \mathrm{m}^{-2} \cdot \mathrm{s}^{-1} \cdot \mathrm{Pa}^{-1}, \mathrm{~N}_{2} / \mathrm{CH}_{4}$ 分离 选择性分别为 $9 、 13$ 。

此外, 针对实际应用场景, 较高硅铝比的 SSZ-13 膜更适合于潮湿环境下的气体分离, 例如: Lee 等 ${ }^{[84]}$ 对比了两种硅铝比 $(\mathrm{Si} / \mathrm{Al}=100 、 \infty)$ 的 SSZ-13 膜在 $\mathrm{CO}_{2} / \mathrm{N}_{2}$ 分离中的性能差异, 发现在干 燥条件下当 $\mathrm{Si} / \mathrm{Al}$ 比从 100 增加到 $\infty$ 时, $\mathrm{CO}_{2} / \mathrm{N}_{2}$ 分离 系数由 4.8 提升至 $~ 6.0$; 在潮湿条件下, $\mathrm{CO}_{2} / \mathrm{N}_{2}$ 分 离系数由 10 提升至 18 。

2)阳离子交换。CHA 膜阳离子种类的交换不仅 能够改变膜表面与气体分子的吸附作用, 还会影响 不同气体的渗透行为, 阳离子的碱性高(电负性低), 与弱酸性分子的结合力越强, 阳离子的电荷密度越 大，与气体的静电作用越强; 另外阳离子交换后, 膜内孔道特征也会发生变化, 从而影响气体分子的 膜内扩散 ${ }^{[85-86]}$ 。Hong 等 ${ }^{[18]}$ 采用 $\mathrm{Li}^{+} 、 \mathrm{Na}^{+} 、 \mathrm{~K}^{+} 、 \mathrm{NH}_{4}^{+}$ 和 $\mathrm{Cu}^{2+}$ 对 H-SAPO-34 膜进行离子交换，其中与碱性 高的 $\mathrm{K}^{+}$交换后, $\mathrm{CO}_{2} / \mathrm{CH}_{4}$ 的分离选择性提升了 $60 \%$, 在所有金属中选择性增强最为显著。Chew 等 ${ }^{\left[{ }^{[99}\right.}$ 利 用不同的碱土阳离子 $\left(\mathrm{Ca}^{2+} 、 \mathrm{Mg}^{2+} 、 \mathrm{Sr}^{2+} 、 \mathrm{Ba}^{2+}\right)$ 与 H-SAPO-34 膜进行交换, 其中与 $\mathrm{Ba}^{2+}$ 交换后的 SAPO-34 膜的 $\mathrm{CO}_{2} / \mathrm{CH}_{4}$ 分离选择性提升约 $240 \%$, 其主要原因在于碱性阳离子增强了酸性 $\mathrm{CO}_{2}$ 分子的 吸附性能(图 4(b))。

如上一节所述, 合成过程中改变阳离子种类可

表 2 SSZ-13 膜和 SAPO-34 膜二次合成优选条件汇总表

Table 2 Summary table of preferred conditions for secondary synthesis of SSZ-13 membrane and SAPO-34 membrane

\begin{tabular}{llll}
\hline \multicolumn{2}{c}{ Influencing factors } & \multicolumn{1}{c}{$\mathrm{SSZ}-13$} & \multicolumn{1}{c}{$\mathrm{SAPO}-34$} \\
\hline $\begin{array}{l}\text { Seed } \\
\text { conditions }\end{array}$ & Support & $\alpha-\mathrm{Al}_{2} \mathrm{O}_{3}$, mullite & $\alpha-\mathrm{Al}_{2} \mathrm{O}_{3}$ \\
& Seed crystal & Ball milled nano seeds & Flake nano seeds \\
& Seeding method & Dip coating & Wipe, electrophoretic deposition \\
Hydrothermal & Formula (structure & Non-pure silica: $1 \mathrm{SiO}_{2}:(5-100) \mathrm{Al}_{2} \mathrm{O}_{3}:(0.1-0.2) \mathrm{NaOH}:$ & $1 \mathrm{Al}_{2} \mathrm{O}_{3}:(1-2) \mathrm{P}_{2} \mathrm{O}_{5}:(0.3-0.6) \mathrm{SiO}_{2}:$ \\
synthesis & directing agent, & $(0-0.06) \mathrm{KOH}(\mathrm{Oriented}$ growth regulation $):$ & $(1-4) \mathrm{TEAOH}:(0-1.6) \mathrm{DPA}:(55-$ \\
conditions & Si/Al, water & $(0.05-0.6) \mathrm{TMAdaOH}:(0-0.05) \mathrm{TEAOH}:$ & $400) \mathrm{H}_{2} \mathrm{O}$ \\
& content, cationic & $(40-120) \mathrm{H}_{2} \mathrm{O}$ & \\
& species) & Pure silica $: 1 \mathrm{SiO}_{2}:(0.5-1.4) \mathrm{TMAdaOH}:$ & $180-230{ }^{\circ} \mathrm{C}$ \\
& & $(0.5-1.4) \mathrm{HF}:(3-6) \mathrm{H}_{2} \mathrm{O}$ & $6-30 \mathrm{~h}$ \\
& Temperature & $160-170{ }^{\circ} \mathrm{C}$ & $400-480{ }^{\circ} \mathrm{C}(4-10 \mathrm{~h})$, temperature \\
& Time & $24-72 \mathrm{~h}$ & rise and fall rate $0.5-2{ }^{\circ} \mathrm{C} / \mathrm{min}$ \\
Calcination & Conventional & $400-550{ }^{\circ} \mathrm{C}(6-12 \mathrm{~h})$, temperature rise and fall rate & $700{ }^{\circ} \mathrm{C} \quad(1-5$ min $)+$ conventional \\
& calcination & $0.2-1{ }^{\circ} \mathrm{C} / \mathrm{min}$ & calcination \\
\hline
\end{tabular}


调节 CHA 膜的取向生长, Sato 等 ${ }^{[55]}$ 通过在合成液中 添加 $\mathrm{K}^{+}$发现在 SSZ-13 膜形成过程中会出现两种形 态, 第一种是沿 $[1,1,1]_{r}$ 轴生长的金字塔形, 第二种 是呈特殊形态的棱镜形; 随着合成时间延长, 第一 种形态逐渐增加, 第二种形态逐渐消失, 从而获得 SSZ-13 膜沿 $[1,1,1]_{r}$ 轴优选取向生长的构型, 使得该 分子篮孔道朝向基本一致, 有助于获得高质量以及 高渗透性的膜。在 $130{ }^{\circ} \mathrm{C}$ 条件下, 针对 $\mathrm{H}_{2} \mathrm{O}(50 \mathrm{wt} \%) /$ NMP $(50 \mathrm{wt} \%)$ 混合体系的分离, 能够得到 1100 的分 离系数和 $36 \mathrm{~kg} \cdot \mathrm{m}^{-2} \cdot \mathrm{h}^{-1}$ 的 $\mathrm{H}_{2} \mathrm{O}$ 渗透通量。

3)杂原子替换。针对 SSZ-13 膜, Ti 原子通过取 代沸石骨架中 $\mathrm{Al}$ 原子实现杂原子替换, 可形成 Ti-CHA 膜, 与高硅或纯硅 CHA 膜相比, Ti-CHA 膜 具有更高的热稳定性、酸稳定性以及疏水性 ${ }^{[87]}$, 从 而促进在含水条件下的气体分离(图 4(c))。Araki 等 ${ }^{[88}$ 发现 $\mathrm{Si} / \mathrm{Ti}$ 比为 347 的 Ti-CHA 膜在 $1150{ }^{\circ} \mathrm{C}$ 热 处理及 $75{ }^{\circ} \mathrm{C}$ 的盐酸溶液处理 $5 \mathrm{~d}$ 后, 其晶体结构仍 能保持不变; 与高硅 $\mathrm{CHA}$ 膜相比, $\mathrm{CO}_{2}$ 的渗透性和 选择性略微降低, 而 $\mathrm{CO}_{2} / \mathrm{CH}_{4}$ 的选择性提高了 1.7 倍至 38。

4)氨基功能化。在 CHA 膜表面接枝氨基官能团, 亦可促进与酸性气体的吸附。针对 SAPO-34 膜, 氨 基基团首先吸附在 B 酸上，桥接 SAPO-34 的-OH 基 团，其他氨基基团可与 SAPO-34 骨架金属原子配位, 其中最易发生配位的是 $\mathrm{Al}-\mathrm{N}^{[89-92]}$ 。含氨基的分子篮 膜能够有效地捕获 $\mathrm{CO}_{2}$, 促进 $\mathrm{CO}_{2}$ 的优先吸附
(图 4(d))。Carreon 等 ${ }^{[36]}$ 通过二胺、己胺和辛胺几种 氨基阳离子接枝到分子䇻骨架中, 通过 FT-IR 光谱 证实了氨基阳离子成功嫁接至 SAPO-34 骨架上; 与 未改性的 SAPO-34 膜相比, 基于乙二胺的氨基功能 化改性的 SAPO-34 膜的 $\mathrm{CO}_{2} / \mathrm{N}_{2}$ 分离系数提高了 $167 \%$ 。

5)表面修饰。利用 $\mathrm{Si}-\mathrm{O}-\mathrm{Si}$ 基团的共价键合，将 硅烷化的咪唑基化合物接枝到 SSZ-13 膜表面，达 到增强膜表面极性的目的, 以促进表面吸附, 但这 种表面修饰也会占据沸石孔口, 使得渗透组份渗透 率降低, 因此需要找到合适的负载量 ${ }^{[93-95]}$ 。周荣飞 等 ${ }^{[28]}$ 利用化学液相沉积法通过沸石表面的羟基与 乙烯基三乙氧基硅烷进行缩合, 以及硅氧烷与 1-烯 丙基咪唑聚合，成功将 $10 \mathrm{wt} \%$ 15wt\%含咪唑基的 硅氧烷聚合物(VTEOS, AMD, VMD) 接枝到 SSZ-13 膜的表面上，其 $\mathrm{CO}_{2} / \mathrm{CH}_{4}$ 选择性(108)提高了 9 倍, $\mathrm{CO}_{2}$ 渗透性 $\left(1.9 \times 10^{-7} \mathrm{~mol} \cdot \mathrm{m}^{-2} \cdot \mathrm{s}^{-1} \cdot \mathrm{Pa}^{-1}\right)$ 仅降低了 $25 \%$ 。 Liu 等 ${ }^{[95}$ 利用膜表面羟基与前驱体之间的硅烷化反 应将 $5 \mathrm{wt} \%$ 硅烷化的咪唑基离子液(RTILs)前驱物接 枝到沸石表面, 使 $\mathrm{CO}_{2} / \mathrm{CH}_{4}$ 的选择性(87)提高了 7 倍, 而 $\mathrm{CO}_{2}$ 的渗透率 $\left(1.0 \times 10^{-7} \mathrm{~mol} \cdot \mathrm{m}^{-2} \cdot \mathrm{s}^{-1} \cdot \mathrm{Pa}^{-1}\right)$ 降低了 $44 \%$ 。

\section{$3.2 \mathrm{CHA}$ 分子篮膜的气体分离应用}

$\mathrm{CHA}$ 分子篮膜在气体分离上表现出极大的应 用潜力, 研究主要集中在 $\mathrm{N}_{2} 、 \mathrm{H}_{2} 、 \mathrm{CO}_{2}$ 以及 $\mathrm{CH}_{4}$ 、 $\mathrm{C}_{2} \mathrm{H}_{6} 、 \mathrm{C}_{3} \mathrm{H}_{8}$ 等轻烃类气体的分离上, 也有对稀有气 体分离等其他体系的探索。图 5 和图 6 分别汇总了
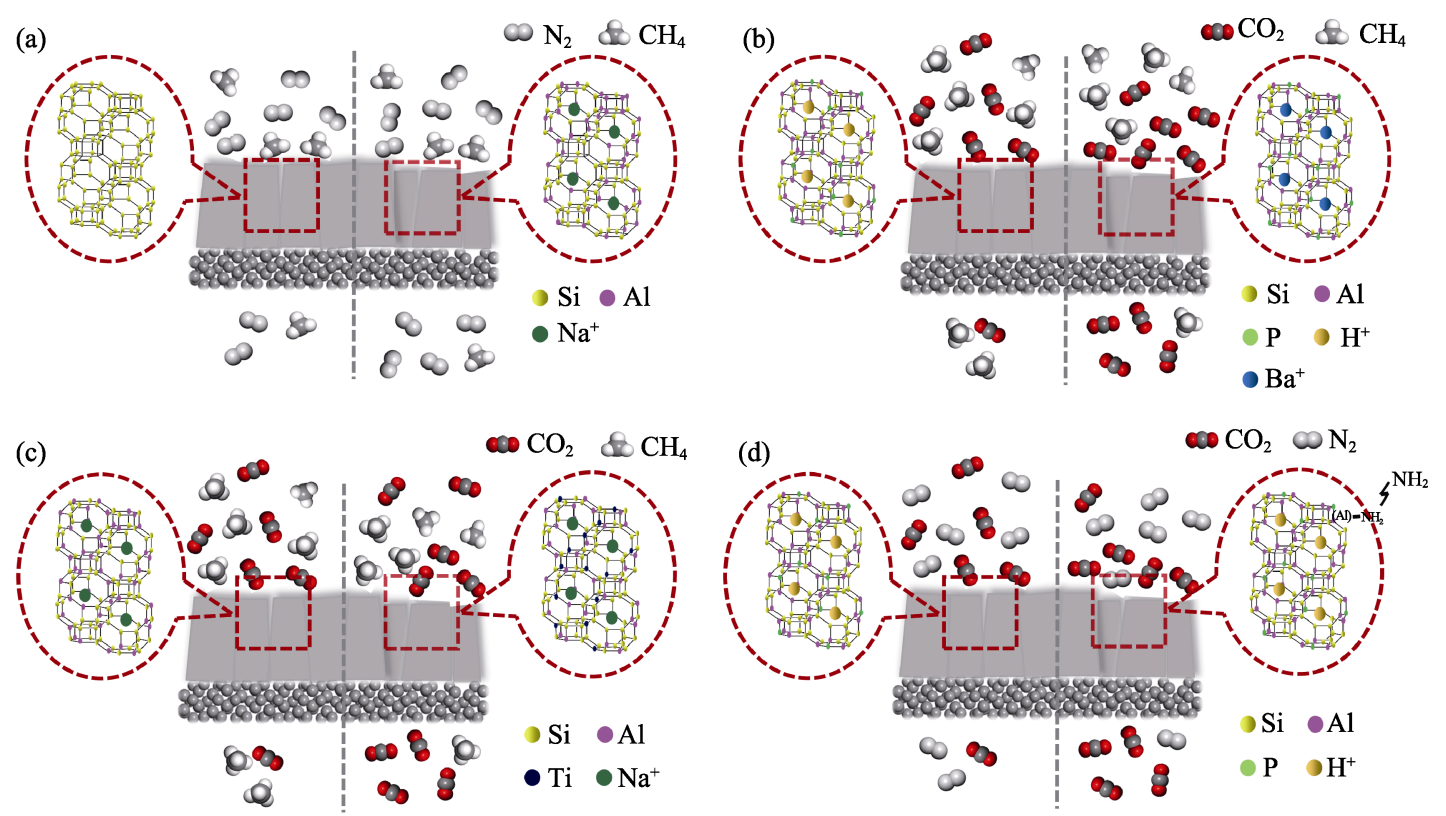

图 4 CHA 分子篮膜表面化学调控前(左)和后(右)的气体分离机制示意图 ${ }^{[24,36,49,83,87]}$

Fig. 4 Schematic diagram of gas separation mechanisms on CHA zeolite membrane before (left) and after (right) the modulation of membrane surface chemistry ${ }^{[24,36,49,83,87]}$

(a) Si/Al regulation; (b) Cation exchange; (c) Heteroatom replacement; (d) Amino functionalization 

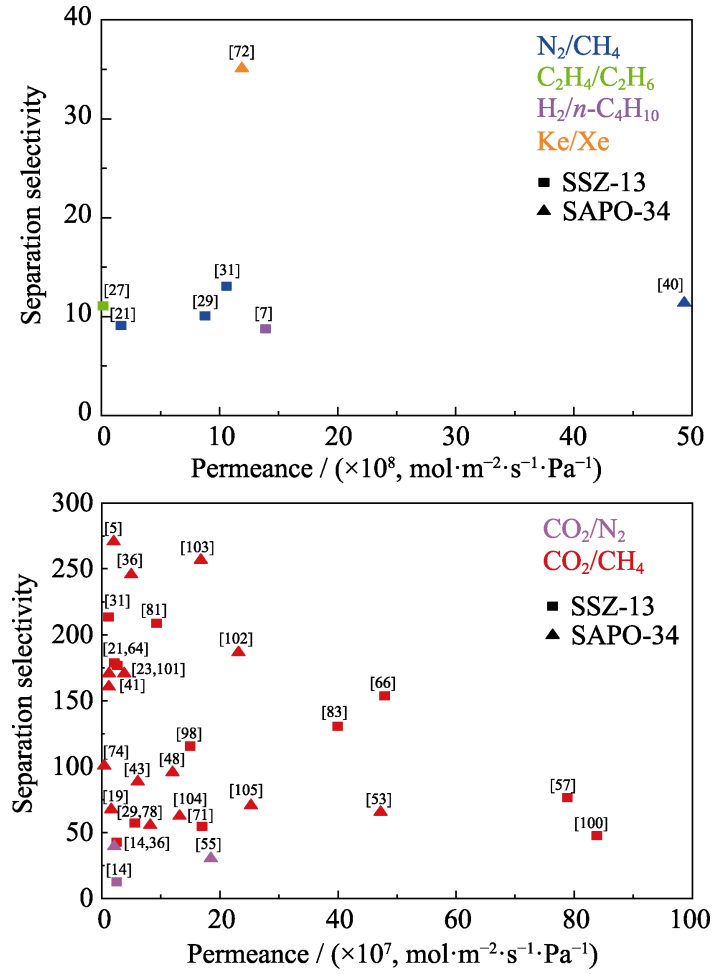

图 $5 \mathrm{CHA}$ 分子篮膜对不同气体体系的分离性能

Fig. 5 Separation performances of different gas mixtures on CHA zeolite membranes

Temperature range: $-25-80{ }^{\circ} \mathrm{C}$; Pressure range: $0.05-0.9 \mathrm{MPa}$

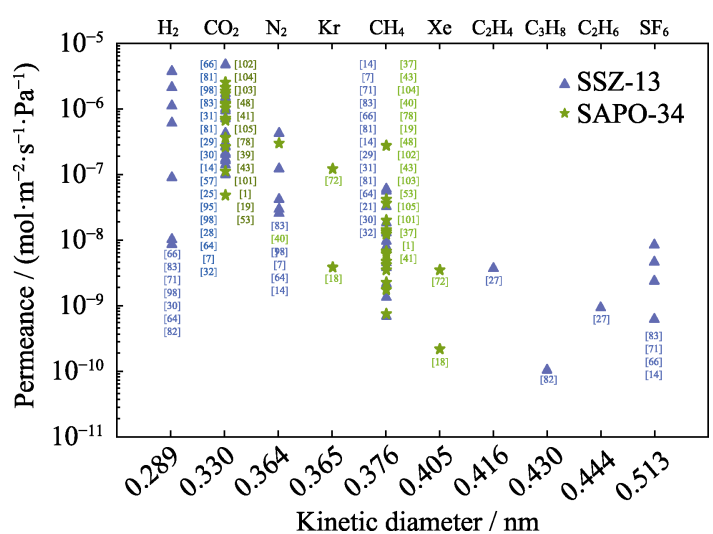

图 6 不同动力学直径气体在 $\mathrm{CHA}$ 分子篎膜的渗透率

Fig. 6 Performance of gas with different kinetic diameters on CHA zeolite membranes

CHA 分子篮膜在各气体分离体系中的分离特性(分 离选择性与渗透率)以及单组分气体的渗透特性(渗
透率)。

对于 $\mathrm{H}_{2} /$ 烷烃体系, 由于二者动力学直径相差 较大，其在 CHA 分子篮膜上的分离性能主要取决 于孔径篎分效应；对于 $\mathrm{Ke} / \mathrm{Xe}$ (核废气回收)、 $\mathrm{C}_{2} \mathrm{H}_{4} / \mathrm{C}_{2} \mathrm{H}_{6}$ (石油精炼)体系, 待分离气体组分的动力 学直径与极化率均相差不大, 其分离性能由表面吸 附与膜内扩散间的具体竞争关系决定。上述气体体 系在 CHA 膜上的分离性能如表 3 所示。

作为三大能源气之一的天然气常常含有 $\mathrm{CO}_{2}$ 、 $\mathrm{N}_{2}$ 等杂质气体 ${ }^{[96]}$, 这些杂质气体的存在不仅会在潮 湿条件下腐蚀运输管道，在使用过程中还会降低天 然气的热值，严重降低天然气的使用效率 ${ }^{[97]}$ 。天然 气中 $\mathrm{CH}_{4}(0.38 \mathrm{~nm}) 、 \mathrm{CO}_{2}(0.33 \mathrm{~nm}) 、 \mathrm{~N}_{2}(0.364 \mathrm{~nm})$ 的 动力学直径均与 $\mathrm{CHA}$ 分子篮膜孔径 $(0.38 \mathrm{~nm})$ 相近, 且 $\mathrm{CH}_{4}\left(25.93 \times 10^{-25} \mathrm{~cm}^{3}, 0\right) 、 \mathrm{CO}_{2}\left(29.11 \times 10^{-25} \mathrm{~cm}^{3}\right.$, $\left.4.30 \times 10^{-26} \mathrm{esu} \cdot \mathrm{cm}^{2}\right) 、 \mathrm{~N}_{2}\left(17.4 \times 10^{-25} \mathrm{~cm}^{3}, 1.52 \times 10^{-26} \mathrm{esu} \cdot \mathrm{cm}^{2}\right)$ 的极化率和四极矩都存在差异，因此，可基于 $\mathrm{CHA}$ 分子篎膜表面吸附与孔径篮分作用对 $\mathrm{CO}_{2} / \mathrm{CH}_{4}$ 、 $\mathrm{CO}_{2} / \mathrm{N}_{2}$ 等体系进行分离, 分离机理如图 6 所示。大 量研究表明通过改变合成条件、优化㷽烧条件、修 饰 $\mathrm{CHA}$ 分子篎膜表面等手段在提高 $\mathrm{CO}_{2}$ 渗透量的 同时也可提高 $\mathrm{CO}_{2} / \mathrm{CH}_{4} 、 \mathrm{CO}_{2} / \mathrm{N}_{2}$ 分离选择性, 具体 分离条件与效果汇总见表 4 。

天然气中除了 $\mathrm{N}_{2} 、 \mathrm{CO}_{2}$ 等一些小分子杂质气体, 还存在一些短链烃类气体 (主要为 $\mathrm{C}_{2} \mathrm{H}_{6} 、 \mathrm{C}_{3} \mathrm{H}_{8}$ 、 $\mathrm{C}_{4} \mathrm{H}_{10}$ ), 虽然浓度很低, 但仍然会影响膜分离, 研究 这些杂质气体对分离体系的干扰程度更有利于控制 实际分离过程。部分学者研究了这些气体对 $\mathrm{CO}_{2} / \mathrm{CH}_{4}$ 分离的影响，如吴婷等 ${ }^{[21]}$ 发现 1\% 9\%的丙 烷会显著降低分离效果, 其对 $\mathrm{N}_{2} / \mathrm{CH}_{4}$ 分离的影响大 于对 $\mathrm{CO}_{2} / \mathrm{CH}_{4}$ 分离的影响, 主要是因为 $\mathrm{CO}_{2}$ 相较于 $\mathrm{N}_{2}$ 与丙烷具有更强的吸附竞争力。Chisholm 等 ${ }^{[22]}$ 利用 SSZ-13 膜从烷烃 $\left(\mathrm{CH}_{4} 、 \mathrm{C}_{3} \mathrm{H}_{8} 、 n-\mathrm{C}_{4} \mathrm{H}_{10}\right)$ 中分离 出 $\mathrm{CO}_{2}$, 发现 $\mathrm{C}_{3} \mathrm{H}_{8}$ 和 $n-\mathrm{C}_{4} \mathrm{H}_{10}$ 虽然吸附在 SSZ-13 晶 体粉末上, 但不吸附在 SSZ-13 膜的孔隙中, 且在气 体混合物中, 相较于 $\mathrm{C}_{2} \mathrm{H}_{6}$ 对 $\mathrm{CO}_{2} / \mathrm{CH}_{4}$ 分离的干扰, 这两种烷烃 $\left(\mathrm{C}_{3} \mathrm{H}_{8} 、 n-\mathrm{C}_{4} \mathrm{H}_{10}\right)$ 对 $\mathrm{CO}_{2}$ 在 SSZ-13 膜上 的渗透能力影响很小。

表 3 CHA 分子篮膜对 $\mathrm{H}_{2}$ 、烃类气体、稀有气体的分离性能

Table 3 Separation performances of $\mathrm{H}_{2}$, hydrocarbon and noble gases on CHA zeolite membranes

\begin{tabular}{clcccccc}
\hline $\begin{array}{c}\text { Serial } \\
\text { number }\end{array}$ & \multicolumn{1}{c}{ Ref. } & Thickness/ $\mu \mathrm{m}$ & Temperature $/{ }^{\circ} \mathrm{C}$ & Pressure/MPa & $\begin{array}{c}\text { Gas separation, } \\
X / Y\end{array}$ & $\begin{array}{c}X \text { permeance/ } \\
\left(\times 10^{-8}, \mathrm{~mol}^{-2} \cdot \mathrm{m}^{-2} \cdot \mathrm{s}^{-1} \cdot \mathrm{Pa}^{-1}\right)\end{array}$ & $\begin{array}{c}\text { Separation } \\
\text { selectivity, } X / Y\end{array}$ \\
\hline 1 & Kalipcilar $^{[7]}$ & $10-40$ & 25 & - & $\mathrm{H}_{2} / n-\mathrm{C}_{4} \mathrm{H}_{10}$ & 14 & 8.7 \\
2 & Zheng $^{[27]}$ & 10 & 30 & 0.2 & $\mathrm{C}_{2} \mathrm{H}_{4} / \mathrm{C}_{2} \mathrm{H}_{6}$ & 0.29 & 11 \\
3 & Feng $^{[72]}$ & 4.3 & 20 & 0.138 & $\mathrm{Kr} / \mathrm{Xe}$ & 12 & 35 \\
4 & Yang $^{[82]}$ & 3.7 & 22 & - & $\mathrm{H}_{2} / \mathrm{C}_{3} \mathrm{H}_{8}$ & 8.4 & 810 \\
\hline
\end{tabular}


表 4 CHA 分子篮膜对 $\mathrm{CO}_{2} / \mathrm{CH}_{4} 、 \mathrm{CO}_{2} / \mathrm{N}_{2} 、 \mathrm{~N}_{2} / \mathrm{CH}_{4} 、 \mathrm{H}_{2} / \mathrm{CH}_{4}$ 分离性能

Table 4 Separation performances of $\mathrm{CO}_{2} / \mathrm{CH}_{4}, \mathrm{CO}_{2} / \mathrm{N}_{2}, \mathrm{~N}_{2} / \mathrm{CH}_{4}, \mathrm{H}_{2} / \mathrm{CH}_{4}$ on $\mathrm{CHA}$ zeolite membranes

\begin{tabular}{|c|c|c|c|c|c|c|c|}
\hline $\begin{array}{c}\text { Serial } \\
\text { number }\end{array}$ & Ref. & Thickness/ $\mu \mathrm{m}$ & Temperature $/{ }^{\circ} \mathrm{C}$ & Pressure/MPa & $\begin{array}{c}\text { Gas separation, } \\
X / Y\end{array}$ & $\begin{array}{c}X \text { permeance/ } \\
\left(\times 10^{-7}, \mathrm{~mol} \cdot \mathrm{m}^{-2} \cdot \mathrm{s}^{-1} \cdot \mathrm{Pa}^{-1}\right)\end{array}$ & $\begin{array}{c}\text { Separation } \\
\text { selectivity, } X / Y\end{array}$ \\
\hline \multirow{2}{*}{1} & \multirow{2}{*}{$\operatorname{Kosinov}^{[14]}$} & \multirow{2}{*}{$4-6$} & 20 & 0.6 & $\mathrm{CO}_{2} / \mathrm{CH}_{4}$ & 2.5 & 42 \\
\hline & & & 20 & 0.6 & $\mathrm{CO}_{2} / \mathrm{N}_{2}$ & 2.5 & 12 \\
\hline \multirow{2}{*}{2} & \multirow{2}{*}{$\mathrm{Wu}^{[21]}$} & \multirow{2}{*}{$6-8$} & 20 & 0.27 & $\mathrm{CO}_{2} / \mathrm{CH}_{4}$ & 2.1 & 178 \\
\hline & & & 20 & 0.27 & $\mathrm{~N}_{2} / \mathrm{CH}_{4}$ & 0.18 & 9 \\
\hline \multirow{2}{*}{3} & \multirow{2}{*}{ Song ${ }^{[29]}$} & \multirow{2}{*}{6} & 25 & 0.2 & $\mathrm{CO}_{2} / \mathrm{CH}_{4}$ & 5.6 & 56.5 \\
\hline & & & 25 & 0.2 & $\mathrm{~N}_{2} / \mathrm{CH}_{4}$ & 0.89 & 10 \\
\hline \multirow{2}{*}{4} & \multirow{2}{*}{$\mathrm{Li}^{[31]}$} & \multirow{2}{*}{2} & 25 & 0.2 & $\mathrm{CO}_{2} / \mathrm{CH}_{4}$ & 1.16 & 213 \\
\hline & & & 25 & 0.2 & $\mathrm{~N}_{2} / \mathrm{CH}_{4}$ & 1.07 & 13 \\
\hline 5 & $\mathrm{Yu}^{[57]}$ & 1.5 & -24 & 0.9 & $\mathrm{CO}_{2} / \mathrm{CH}_{4}$ & 79 & 76 \\
\hline 6 & Karakiliç ${ }^{[64]}$ & $2-4$ & 22 & 0.2 & $\mathrm{CO}_{2} / \mathrm{CH}_{4}$ & 2.6 & 176 \\
\hline 7 & $\mathrm{Qiu}^{[66]}$ & 0.44 & 20 & 0.14 & $\mathrm{CO}_{2} / \mathrm{CH}_{4}$ & 48 & 153 \\
\hline \multirow{2}{*}{8} & \multirow{2}{*}{$\operatorname{Kida}^{[71]}$} & \multirow{2}{*}{-} & 40 & 0.1 & $\mathrm{CO}_{2} / \mathrm{CH}_{4}$ & 17 & 54 \\
\hline & & & 40 & 0.1 & $\mathrm{H}_{2} / \mathrm{CH}_{4}$ & 11 & 34 \\
\hline 9 & $\operatorname{Tang}^{[81]}$ & 10 & 20 & 0.2 & $\mathrm{CO}_{2} / \mathrm{CH}_{4}$ & 9.3 & 208 \\
\hline 10 & $\mathrm{Kida}^{[83]}$ & 5 & 25 & 0.1 & $\mathrm{CO}_{2} / \mathrm{CH}_{4}$ & 40 & 130 \\
\hline 11 & Imasaka $^{[98]}$ & 3 & 40 & 0.3 & $\mathrm{CO}_{2} / \mathrm{CH}_{4}$ & 15 & 115 \\
\hline 12 & Maghsoudi ${ }^{[99]}$ & 20 & 30 & 0.1 & $\mathrm{CO}_{2} / \mathrm{CH}_{4}$ & 0.34 & 21.6 \\
\hline 13 & $\mathrm{Yu}^{[100]}$ & 1.3 & 3 & 0.9 & $\mathrm{CO}_{2} / \mathrm{CH}_{4}$ & 84 & 47 \\
\hline 14 & $\mathrm{Li}^{[5]}$ & 5 & 80 & 0.14 & $\mathrm{CO}_{2} / \mathrm{CH}_{4}$ & 2.0 & 270 \\
\hline 15 & $\mathrm{Li}^{[19]}$ & - & 24 & 0.138 & $\mathrm{CO}_{2} / \mathrm{CH}_{4}$ & 1.6 & 67 \\
\hline 16 & Carreon $^{[23]}$ & - & 22 & 0.138 & $\mathrm{CO}_{2} / \mathrm{CH}_{4}$ & 3.8 & 170 \\
\hline \multirow{2}{*}{17} & \multirow{2}{*}{ Venna $^{[36]}$} & \multirow{2}{*}{-} & 22 & 0.138 & $\mathrm{CO}_{2} / \mathrm{CH}_{4}$ & 5.0 & 245 \\
\hline & & & 22 & 0.138 & $\mathrm{CO}_{2} / \mathrm{N}_{2}$ & 2.1 & 39 \\
\hline 18 & Huang $^{[40]}$ & 2 & 22 & 0.074 & $\mathrm{~N}_{2} / \mathrm{CH}_{4}$ & 4.93 & 11.3 \\
\hline 19 & Chen $^{[41]}$ & $2-3$ & 25 & 0.1 & $\mathrm{CO}_{2} / \mathrm{CH}_{4}$ & 1.18 & 160 \\
\hline 20 & Chang $^{[43]}$ & - & - & 4 & $\mathrm{CO}_{2} / \mathrm{CH}_{4}$ & 6.1 & 88 \\
\hline 21 & $\mathrm{Liu}^{[48]}$ & 3 & 30 & 0.1 & $\mathrm{CO}_{2} / \mathrm{CH}_{4}$ & 12 & 95 \\
\hline 22 & $\operatorname{Rehman}^{[53]}$ & - & 80 & 0.4 & $\mathrm{CO}_{2} / \mathrm{CH}_{4}$ & 47.3 & 65 \\
\hline 23 & $\operatorname{Liu}^{[55]}$ & $7-15$ & 25 & 0.1 & $\mathrm{CO}_{2} / \mathrm{N}_{2}$ & 18.5 & 29.8 \\
\hline 24 & $\mathrm{Li}^{[74]}$ & $4-6$ & 22 & 7 & $\mathrm{CO}_{2} / \mathrm{CH}_{4}$ & 0.4 & 100 \\
\hline 25 & Zhang $^{[78]}$ & - & 20 & 4.6 & $\mathrm{CO}_{2} / \mathrm{CH}_{4}$ & 8.2 & 55 \\
\hline 26 & Noble $^{[101]}$ & - & 22 & 0.14 & $\mathrm{CO}_{2} / \mathrm{CH}_{4}$ & 1.2 & 170 \\
\hline 27 & $\mathrm{Shi}^{[102]}$ & $2-4$ & 22 & 0.14 & $\mathrm{CO}_{2} / \mathrm{CH}_{4}$ & 23.2 & 186 \\
\hline 28 & $\mathrm{Shi}^{[103]}$ & $4-5$ & 22 & 0.14 & $\mathrm{CO}_{2} / \mathrm{CH}_{4}$ & 16.8 & 256 \\
\hline 29 & $\mathrm{Li}^{[104]}$ & 3 & - & 4 & $\mathrm{CO}_{2} / \mathrm{CH}_{4}$ & 13.2 & 62 \\
\hline 30 & $\mathrm{Bai}^{[105]}$ & 0.8 & - & 0.2 & $\mathrm{CO}_{2} / \mathrm{CH}_{4}$ & 25.3 & 70 \\
\hline
\end{tabular}

\section{4 结语与展望}

本文立足于小孔分子篮 CHA 膜的合成与气体 分离应用, 介绍了目前合成 $\mathrm{CHA}$ 分子笁膜的常用 方法, 特别是探讨了主流二次合成法中关键合成条 件对薄膜质量的影响规律; 总结归纳了 CHA 分子 膜对轻质气体分离的性能以及应用潜能, 着重研究 了气体分离增强策略, 为今后功能导向的分子篎膜
的设计合成与开发提供了重要的科学参考。

经过 20 年的发展, CHA 分子篮膜在制备与气体 分离方面取得了一定进展, 然而 CHA 膜在实际应 用方面仍有巨大的提升空间。基于当前主流的二次 生长法, CHA 分子篮膜的实验室级小规模合成已日 趋成熟, 在合成条件与操作影响因素较多的情况下 能实现部分性能的精准调控, 其中控制膜厚、晶体 取向生长、膜缺陷等调控值得继续深入探究。 
为了实现工业应用, $\mathrm{CHA}$ 分子篎膜的批量化制备势 在必行, 虽然已出现相关案例, 但仍然存在成本昂 贵、良品率不足等问题。昂贵的模板剂和载体 ${ }^{[106]}$ 是 CHA 分子篎膜生产成本控制的重点, 已有研究 者提出采用廉价模板剂合成 CHA 分子篎膜, 但膜 质量暂时还难以达到要求, 未来需深入探索经济型 模板剂降低成本; 目前常用的 $\alpha$-氧化铝与莫来石载 体也难以避免成本较高的问题, 因此寻找或开发一 种价格低廉且性能与 CHA 分子篮膜相适配的载体 或是未来的重要课题之一。

在 CHA 分子篎膜的其他制备方法方面, 微波 加热法有望成为新兴的膜合成思路, 虽然当前该法 存在设备成本较高以及运行能耗高等问题，但其合 成时间短以及成功率高等优势，使其在合成时间 长、难以控制膜厚的 $\mathrm{CHA}$ 分子篮膜的制备上具有 较大的潜力, 值得进一步研究, 重点需要解决微波 合成设备放大与运行能耗降低等问题。

在 CHA 分子篎膜的气体分离方面，目前研究 大多集中在天然气、氢气等能源气体回收，少量用 于核废料稀有气体回收。考虑到 CHA 膜适宜的孔 道尺寸与丰富可调的表面特性, 其能覆盖到的气体 分离体系应不限于此，未来可深入探索其他工业气 体体系与 CHA 分子篎膜的匹配性, 扩充其气体分 离应用场合。例如, 光纤制造中 $\mathrm{He} / \mathrm{O}_{2}$ 的分离用于 $\mathrm{He}$ 回收、 $\mathrm{D}_{2} / \mathrm{N}_{2}$ 分离用于 $\mathrm{D}_{2}$ 回收、烟气解吸气中 $\mathrm{SO}_{2} / \mathrm{NO}_{2}$ 的分离用于二者富集资源化等。

\section{参考文献:}

[1] LI S, CARREON M A, ZHANG Y, et al. Scale-up of SAPO-34 membranes for $\mathrm{CO}_{2} / \mathrm{CH}_{4}$ separation. Journal of Membrane Science, 2010, 352(1/2): 7-13.

[2] NAIR S, LAI Z, NIKOLAKIS V, et al. Separation of closeboiling hydrocarbon mixtures by MFI and FAU membranes made by secondary growth. Microporous \& Mesoporous Materials, 2001, 48(1): 219-228.

[3] GU X H, DONG J H, NENOFF T M, et al. Synthesis of defect-free FAU-type zeolite membranes and separation for dry and moist $\mathrm{CO}_{2} / \mathrm{N}_{2}$ mixtures. Industrial \& Engineering Chemistry Research, 2005, 44(4): 937-944.

[4] ZHU W, GORA L, VAN DEN BERG A W C, et al. Water vapour separation from permanent gases by a zeolite-4A membrane. Journal of Membrane Science, 2005, 253(1/2): 57-66.

[5] LI S, MARTINEK J G, FALCONER J L, et al. High-pressure $\mathrm{CO}_{2} / \mathrm{CH}_{4}$ separation using SAPO-34 membranes. Industrial \& Engineering Chemistry Research, 2005, 44(9): 3220-3228.

[6] MA L, CHENG Y, CAVATAIO G, et al. Characterization of commercial $\mathrm{Cu}-\mathrm{SSZ}-13$ and $\mathrm{Cu}-\mathrm{SAPO}-34$ catalysts with hydrothermal treatment for $\mathrm{NH}_{3}$-SCR of $\mathrm{NO}_{x}$ in diesel exhaust. Chemical Engineering Journal, 2013, 225(3): 323-330.

[7] KALIPCILAR H, BOWEN T C, NOBLE R D, et al. Synthesis and separation performance of SSZ-13 zeolite membranes on tubular supports. Chemistry of Materials, 2002, 14(8): 3458-3464.

[8] ZHANG L X, JIA M D, MIN E. Synthesis of SAPO-34/ceramic composite membranes. Studies in Surface Science and Catalysis,
1997, 105(11): 2211-2216.

[9] YAN N, XU H, ZHANG W, et al. Probing locations of organic structure-directing agents (OSDAs) and host-guest interactions in CHA-type SAPO-34/44. Microporous and Mesoporous Materials, 2018, 264: 55-59.

[10] YU M, NOBLE R D, FALCONER J L. Zeolite membranes: microstructure characterization and permeation mechanisms. Accounts of Chemical Research, 2011, 44(11): 1196-1206.

[11] WANG J Q, YANG J H, LI H Z, et al. Research progress of zeolite molecular sieve membrane. Membrane Science and Technology, 2014, 34(3): 1-7, 42 .

[12] CARO J, NOACK M. Zeolite membranes-recent developments and progress. Microporous and Mesoporous Materials, 2008, 115(3): 215-233.

[13] BOWEN T C, NOBLE R D, FALCONER J L. Fundamentals and applications of pervaporation through zeolite membranes. Journal of Membrane Science, 2004, 245(1/2): 1-33.

[14] KOSINOV N, AUFFRET C, GVCVCYENER C, et al. High flux high-silica SSZ-13 membrane for $\mathrm{CO}_{2}$ separation. Journal of Materials Chemistry A, 2014, 2(32): 13083-13092.

[15] TOTH A J, SZILAGYI B, HAAZ E, et al. Enhanced separation of maximum boiling azeotropic mixtures with extractive heterogeneous-azeotropic distillation. Chemical Engineering Research and Design, 2019, 147: 55-62.

[16] CLET G, GORA L, NISHIYAMA N, et al. An alternative synthesis method for zeolite Y membranes. Chemical Communications, 2001, 1: 41-42.

[17] JIANG H, ZHANG B, LIN Y S, et al. Synthesis of zeolite membranes. Chinese Science Bulletin, 2004, 49(24): 2547-2554.

[18] HONG M, LI S, FUNKE H F, et al. Ion-exchanged SAPO-34 membranes for light gas separations. Microporous and Mesoporous Materials, 2007, 106(1/2/3): 140-146.

[19] LI S, FALCONER J L, NOBLE R D. SAPO-34 membranes for $\mathrm{CO}_{2} / \mathrm{CH}_{4}$ separation. Journal of Membrane Science, 2004, 241(1): 121-135.

[20] SAPATSIS M, LOVALLO M, OKUBO T, et al. Characterization of zeolite L nanoclusters. Chemistry of Materials, 1995, 7(9): 1734-1741.

[21] WU T, DIAZ M C, ZHENG Y, et al. Influence of propane on $\mathrm{CO}_{2} / \mathrm{CH}_{4}$ and $\mathrm{N}_{2} / \mathrm{CH}_{4}$ separations in CHA zeolite membranes. Journal of Membrane Science, 2015, 473: 201-209.

[22] CHISHOLM N O, FUNKE H H, NOBLE R D, et al. Carbon dioxide/alkane separations in a SSZ-13 membrane. Journal of Membrane Science, 2018, 568: 17-21.

[23] CARREON M A, LI S, FALCONER J L, et al. SAPO-34 seeds and membranes prepared using multiple structure directing agents. Advanced Materials, 2008, 20(4): 729-732.

[24] KOSINOV N, AUFFRET C, BORGHUIS G J, et al. Influence of the $\mathrm{Si} / \mathrm{Al}$ ratio on the separation properties of SSZ-13 zeolite membranes. Journal of Membrane Science, 2015, 484: 140-145.

[25] LEE M, HONG S, KIM D, et al. Chabazite-type zeolite membranes for effective $\mathrm{CO}_{2}$ separation: the role of hydrophobicity and defect structure. Applied Materials \& Interfaces, 2019, 11(4): 3946-3960.

[26] HONG S, KIM D, JEONG Y, et al. Healing of microdefects in SSZ-13 membranes via filling with dye molecules and its effect on dry and wet $\mathrm{CO}_{2}$ separations. Chemistry of Materials, 2018, 30(10): 3346-3358.

[27] ZHENG Y, HU N, WANG H, et al. Preparation of steam-stable high-silica CHA (SSZ-13) membranes for $\mathrm{CO}_{2} / \mathrm{CH}_{4}$ and $\mathrm{C}_{2} \mathrm{H}_{4} / \mathrm{C}_{2} \mathrm{H}_{6}$ separation. Journal of Membrane Science, 2015, 475: 303-310.

[28] ZHOU R, WANG H, WANG B, et al. Defect-patching of zeolite membranes by surface modification using siloxane polymers for $\mathrm{CO}_{2}$ separation. Industrial \& Engineering Chemistry Research, 2015, 54(30): 7516-7523.

[29] SONG S, GAO F, ZHANG Y, et al. Preparation of SSZ-13 membranes with enhanced fluxes using asymmetric alumina supports for $\mathrm{N}_{2} / \mathrm{CH}_{4}$ and $\mathrm{CO}_{2} / \mathrm{CH}_{4}$ separations. Separation and 
Purification Technology, 2019, 209: 946-954.

[30] WANG B, ZHENG Y, ZHANG J, et al. Separation of light gas mixtures using zeolite SSZ-13 membranes. Microporous and Mesoporous Materials, 2019, 275: 191-199.

[31] LI X, WANG Y, WU T, et al. High-performance SSZ-13 membranes prepared using ball-milled nanosized seeds for carbon dioxide and nitrogen separations from methane. Chinese Journal of Chemical Engineering, 2020, 28(5): 1285-1292.

[32] LIANG L, ZHU M, CHEN L, et al. Single gas permeance performance of high silica SSZ-13 zeolite membranes. Membranes, 2018, 8(3): 43.

[33] JIANG J, WANG X, ZHANG Y, et al. Fabrication of pure-phase CHA zeolite membranes with ball-milled seeds at low $\mathrm{K}^{+}$ concentration. Microporous and Mesoporous Materials, 2015, 215: $98-108$.

[34] JIANG J, PENG L, WANG X, et al. Effect of Si/Al ratio in the framework on the pervaporation properties of hollow fiber CHA zeolite membranes. Microporous and Mesoporous Materials, 2019, 273: 196-202.

[35] WU T, LUCERO J, CRAWFORD J M, et al. SAPO-34 membranes for xenon capture from air. Journal of Membrane Science, 2019, 573: 288-292.

[36] VENNA S R, CARREON M A. Amino-functionalized SAPO-34 membranes for $\mathrm{CO}_{2} / \mathrm{CH}_{4}$ and $\mathrm{CO}_{2} / \mathrm{N}_{2}$ separation. Langmuir, 2011, 27(6): 2888-2894.

[37] ZONG Z, CARREON M A. Thin SAPO-34 membranes synthesized in stainless steel autoclaves for $\mathrm{N}_{2} / \mathrm{CH}_{4}$ separation. Journal of Membrane Science, 2017, 524: 117-123.

[38] FUNKE H H, TOKAY B, ZHOU R, et al. Spatially resolved gas permeation through SAPO-34 membranes. Journal of Membrane Science, 2012, 409: 212-221.

[39] PING E W, ZHOU R, FUNKE H H, et al. Seeded-gel synthesis of SAPO-34 single channel and monolith membranes, for $\mathrm{CO}_{2} / \mathrm{CH}_{4}$ separations. Journal of Membrane Science, 2012, 415: 770-775.

[40] HUANG Y, WANG L, SONG Z, et al. Growth of high-quality, thickness-reduced zeolite membranes towards $\mathrm{N}_{2} / \mathrm{CH}_{4}$ separation using high-aspect-ratio seeds. Angewandte Chemie International Edition, 2015, 54(37): 10843-10847.

[41] CHEN Y, ZHANG Y, ZHANG C, et al. Fabrication of high-flux SAPO-34 membrane on $\alpha-\mathrm{Al}_{2} \mathrm{O}_{3}$ four-channel hollow fibers for $\mathrm{CO}_{2}$ capture from $\mathrm{CH}_{4}$. Journal of $\mathrm{CO}_{2}$ Utilization, 2017, 18: 30-40.

[42] WANG M, LI M, CHANG N, et al. Vapor separation of methanol-dimethyl carbonate mixture on SAPO-34 zeolite membrane. Journal of Membrane Science, 2018, 565: 311-321.

[43] CHANG N, TANG H, BAI L, et al. Optimized rapid thermal processing for the template removal of SAPO-34 zeolite membranes. Journal of Membrane Science, 2018, 552: 13-21.

[44] LightFoOT P, WOODCOCK D A, MAPLE M J, et al. The widespread occurrence of negative thermal expansion in zeolites. Journal of Materials Chemistry, 2001, 11(1): 212-216.

[45] SATO K, SUGIMOTO K, SHIMOTSUMA N, et al. Development of practically available up-scaled high-silica CHA-type zeolite membranes for industrial purpose in dehydration of N-methyl pyrrolidone solution. Journal of Membrane Science, 2012, 409: 82-95.

[46] BRAUN I, SCHULZ EKLOFF G, WOHRLE D, et al. Synthesis of $\mathrm{AlPO}_{4}-5$ in a microwave-heated, continuous-flow, highpressure tube reactor. Microporous and Mesoporous Materials, 1998, 23(1/2): 79-81.

[47] HU N, LI Y, ZHONG S, et al. Microwave synthesis of zeolite CHA (chabazite) membranes with high pervaporation performance in absence of organic structure directing agents. Microporous and Mesoporous Materials, 2016, 228: 22-29.

[48] LIU X, DU S, ZHANG B. The seeded growth of dense and thin SAPO-34 membranes on porous $\alpha-\mathrm{Al}_{2} \mathrm{O}_{3}$ substrates under microwave irradiation. Materials Letters, 2013, 91: 195-197.
[49] CHEW T L, AHMAD A L, BHATIA S. Ba-SAPO-34 membrane synthesized from microwave heating and its performance for $\mathrm{CO}_{2} / \mathrm{CH}_{4}$ gas separation. Chemical Engineering Journal, 2011, 171(3): 1053-1059.

[50] AKHTAR F, OJUVA A, WIRAWAN S K, et al. Hierarchically porous binder-free silicalite-1 discs: a novel support for allzeolite membranes. Journal of Materials Chemistry, 2011, 21(24): 8822-8828.

[51] HE Y, CUI X, LIU X, et al. Preparation of self-supporting NaA zeolite membranes using geopolymers. Journal of Membrane Science, 2013, 447: 66-72.

[52] STOEGER J A, CHOI J, TSAPATSIS M. Rapid thermal processing and separation performance of columnar MFI membranes on porous stainless steel tubes. Energy \& Environmental Science, 2011, 4(9): 3479-3486.

[53] REHMAN R U, SONG Q, PENG L, et al. Hydrophobic modification of SAPO-34 membranes for improvement of stability under wet condition. Chinese Journal of Chemical Engineering, 2019, 27(10): 2397-2406.

[54] MU Y, CHEN H, XIANG H, et al. Defects-healing of SAPO-34 membrane by post-synthesis modification using organosilica for selective $\mathrm{CO}_{2}$ separation. Journal of Membrane Science, 2019, 575: 80-88.

[55] LIU B, TANG C, LI X, et al. High-performance SAPO-34 membranes for $\mathrm{CO}_{2}$ separations from simulated flue gas. Microporous and Mesoporous Materials, 2020, 292: 109712.

[56] KOSINOV N, AUFFRET C, SRITAPHI V G P, et al. Influence of support morphology on the detemplation and permeation of ZSM-5 and SSZ-13 zeolite membranes. Microporous and Mesoporous Materials, 2014, 197: 268-277.

[57] YU L, HOLMGREN A, HEDLUND J. A novel method for fabrication of high-flux zeolite membranes on supports with arbitrary geometry. Journal of Materials Chemistry A, 2019, 7(17): 10325-10330.

[58] CARREON M A, LI S, FALCONER J L, et al. Aluminasupported SAPO-34 membranes for $\mathrm{CO}_{2} / \mathrm{CH}_{4}$ separation. Journal of the American Chemical Society, 2008, 130(16): 5412-5413.

[59] LIU J Q, LUO Y B, LI M G, et al. Synthesis of nanosized SSZ-13 zeolite and performance of its mixed matrix membrane for $\mathrm{CO}_{2} / \mathrm{CH}_{4}$ separation. China Petroleum Processing \& Petrochemical Technology, 2019, 21(2): 19-26.

[60] TAKATA T, TSUNOJI N, TAKAMITSU Y, et al. Nanosized CHA zeolites with high thermal and hydrothermal stability derived from the hydrothermal conversion of FAU zeolite. Microporous and Mesoporous Materials, 2016, 225: 524-533.

[61] NAJAFI N, ASKARI S, HALLADJ R. Hydrothermal synthesis of nanosized SAPO-34 molecular sieves by different combinations of multi templates. Powder Technology, 2014, 254: 324-330.

[62] YANG H, LIU X, LU G, et al. Synthesis of SAPO-34 nanoplates via hydrothermal method. Microporous and Mesoporous Materials, 2016, 225: 144-153.

[63] SUN Q, WANG N, GUO G, et al. Ultrafast synthesis of nanosized zeolite SAPO-34 with excellent MTO catalytic performance. Chemical Communications, 2015, 51(91): 16397-16400.

[64] KARAKILIÇ P, WANG X, KAPTEIJN F, et al. Defect-free high-silica CHA zeolite membranes with high selectivity for light gas separation. Journal of Membrane Science, 2019, 586: 34-43.

[65] JABBARI Z, FATEMI S, DAVOODPOUR M. Comparative study of seeding methods; dip-coating, rubbing and EPD, in SAPO-34 thin film fabrication. Advanced Powder Technology, 2014, 25(1): 321-330.

[66] HENGE Q, ZHANG Y, KONG L, et al. High performance SSZ-13 membranes prepared at low temperature. Journal of Membrane Science, 2020, 603: 118023.

[67] WHITE J C, DUTTA P K, SHQAU K, et al. Synthesis of ultrathin zeolite $Y$ membranes and their application for separation of carbon dioxide and nitrogen gases. Langmuir, 2010, 26(12): 10287-10293. 
[68] BOHSTRÖM Z, ARSTAD B, LILLERUD K P. Preparation of high silica chabazite with controllable particle size. Microporous and Mesoporous Materials, 2014, 195: 294-302.

[69] VAN HEYDEN H, MINTOVA S, BEIN T. Nanosized SAPO-34 synthesized from colloidal solutions. Chemistry of Materials, 2008, 20(9): 2956-2963.

[70] MIYAMOTO M, NAKATANI T, FUJIOKA Y, et al. Verified synthesis of pure silica CHA-type zeolite in fluoride media. Microporous and Mesoporous Materials, 2015, 206: 67-74.

[71] KIDA K, MAETA Y, YOGO K. Preparation and gas permeation properties on pure silica CHA-type zeolite membranes. Journal of Membrane Science, 2017, 522: 363-370.

[72] FENG X, ZONG Z, ELSAIDIl S K, et al. $\mathrm{Kr} / \mathrm{Xe}$ separation over a chabazite zeolite membrane. Journal of the American Chemical Society, 2016, 138(31): 9791-9794.

[73] CARREON M A. Molecular sieve membranes for $\mathrm{N}_{2} / \mathrm{CH}_{4}$ separation. Journal of Materials Research, 2018, 33(1): 32-43.

[74] LI S, FALCONER J L, NOBLE R D. SAPO-34 membranes for $\mathrm{CO}_{2} / \mathrm{CH}_{4}$ separations: effect of $\mathrm{Si} / \mathrm{Al}$ ratio. Microporous and Mesoporous Materials, 2008, 110(2/3): 310-317.

[75] KIM S J, LIU Y, MOORE J S, et al. Thin hydrogen-selective SAPO-34 zeolite membranes for enhanced conversion and selectivity in propane dehydrogenation membrane reactors. Chemistry of Materials, 2016, 28(12): 4397-4402.

[76] RIVERA RAMOS M E, RUIZ MERCADO G J, HERNANDEZ MALDONADO A J. Separation of $\mathrm{CO}_{2}$ from light gas mixtures using ion-exchanged silicoaluminophosphate nanoporous sorbents. Industrial \& Engineering Chemistry Research, 2008, 47(15): 5602-5610.

[77] AVILA A M, FUNKE H H, ZHANG Y, et al. Concentration polarization in SAPO-34 membranes at high pressures. Journal of Membrane Science, 2009, 335(1/2): 32-36.

[78] ZHANG Y, TOKAY B, FUNKE H H, et al. Template removal from SAPO-34 crystals and membranes. Journal of Membrane Science, 2010, 363(1/2): 29-35.

[79] PENG C, LIU Z, HOORIMOTO A, et al. Preparation of nanosized SSZ-13 zeolite with enhanced hydrothermal stability by a two-stage synthetic method. Microporous and Mesoporous Materials, 2018, 255: 192-199.

[80] KIM J, JANG E, HONG S, et al. Microstructural control of a SSZ-13 zeolite film via rapid thermal processing. Journal of Membrane Science, 2019, 591: 117342.

[81] TANG H, BAI L, WANG M, et al. Fast synthesis of thin high silica SSZ-13 zeolite membrane using oil-bath heating. International Journal of Hydrogen Energy, 2019, 44(41): 23107-23119.

[82] YANG S, KWON Y H, KOH D Y, et al. Highly selective SSZ-13 zeolite hollow fiber membranes by ultraviolet activation at near-ambient temperature. ChemNanoMat, 2019, 5(1): 61-67.

[83] KIDA K, MAETA Y, YOGO K. Pure silica CHA-type zeolite membranes for dry and humidified $\mathrm{CO}_{2} / \mathrm{CH}_{4}$ mixtures separation. Separation and Purification Technology, 2018, 197: 116-121.

[84] LEE M, JEONG Y, HONG S, et al. High performance $\mathrm{CO}_{2}$-perm-selective SSZ-13 membranes: elucidation of the link between membrane material and module properties. Journal of Membrane Science, 2020, 611: 118390.

[85] DJIEUGOUE M A, PRAKASH A M, KEVAN L. Catalytic study of methanol-to-olefins conversion in four small-pore silicoaluminophosphate molecular sieves: influence of the structural type, nickel incorporation, nickel location, and nickel concentration. Journal of Physical Chemistry B, 2000, 104(27): 6452-6461.

[86] CHEN X, GUO J, FU Z, et al. Characterization and catalytic behaviors of methylamine modified FAU zeolites. Journal of Porous Materials, 2013, 20(5):1271-1281.

[87] IMASAKA, ISHII H, HAYASHI J, et al. Synthesis of CHA-type titanosilicate zeolites using titanium oxide as Ti source and evaluation of their physicochemical properties. Microporous and Mesoporous Materials, 2019, 273: 243-248.

[88] ARAKI S, ISHII H, IMASAKA S, et al. Synthesis and gas permeation properties of chabazite-type titanosilicate membranes synthesized using nano-sized seed crystals. Microporous and Mesoporous Materials, 2020, 292: 109798.

[89] SERGEI A ZUBKOV, LEONID M KUSTOV, VADIM B KAZANSKY, et al. Investigation of hydroxyl groups in crystalline silicoaluminophosphate SAPO-34 by diffuse reflectance infrared spectroscopy. Journal of the Chemical Society, 1991, 87: 897.

[90] HUANG J, ZOU J, HO W S W. Carbon dioxide capture using a CO-selective facilitated transport membrane. Industrial \& Engineering Chemistry Research, 2008, 47(4): 1261-1267.

[91] ZOU J, $\mathrm{HO} \mathrm{W} \mathrm{S} \mathrm{W.} \mathrm{CO}_{2}$-selective polymeric membranes containing amines in crosslinked poly(vinyl alcohol). Journal of Membrane Science, 2006, 286(1/2): 310-321.

[92] TEE Y H, ZOU J, HO W S W. $\mathrm{CO}_{2}$-selective membranes containing dimethylglycine mobile carriers and polyethylenimine fixed carrier. Journal of the Chinese Institute of Chemical Engineers, 2006, 37(1): 37-47.

[93] SINGH Z V, COWAN M G, MCDANEL W M, et al. Determination and optimization of factors affecting $\mathrm{CO}_{2} / \mathrm{CH}_{4}$ separation performance in poly(ionic liquid)-ionic liquid-zeolite mixed-matrix membranes. Journal of Membrane Science, 2016, 509: $149-155$.

[94] BARA J E, CAMPER D E, GIN D L, et al. Room-temperature ionic liquids and composite materials: platform technologies for $\mathrm{CO}_{2}$ capture. Accounts of Chemical Research, 2010, 43(1): $152-159$.

[95] LIU B, ZHOU R, BU N, et al. Room-temperature ionic liquids modified zeolite SSZ-13 membranes for $\mathrm{CO}_{2} / \mathrm{CH}_{4}$ separation. Journal of Membrane Science, 2017, 524: 12-19.

[96] CHEN K J, MADDEN D G, PHAM T, et al. Tuning pore size in square-lattice coordination networks for size-selective sieving of $\mathrm{CO}_{2}$. Angewandte Chemie International Edition, 2016, 55(35): 10268-10272.

[97] SINGH Z V, COWAN M G, MCDANEL W M, et al. Determination and optimization of factors affecting $\mathrm{CO}_{2} / \mathrm{CH}_{4}$ separation performance in poly (ionic liquid)-ionic liquid-zeolite mixed-matrix membranes. Journal of Membrane Science, 2016, 509: 149-155.

[98] IMASAKA S, ITAKURA M, YANO K, et al. Rapid preparation of high-silica CHA-type zeolite membranes and their separation properties. Separation and Purification Technology, 2018, 199: 298-303.

[99] MAGHSOUDI H, SOLTANIEH M. Simultaneous separation of $\mathrm{H}_{2} \mathrm{~S}$ and $\mathrm{CO}_{2}$ from $\mathrm{CH}_{4}$ by a high silica CHA-type zeolite membrane. Journal of Membrane Science, 2014, 470: 159-165.

[100] YU L, HOLMGREN A, ZHOU M, et al. Highly permeable CHA membranes prepared by fluoride synthesis for efficient $\mathrm{CO}_{2} / \mathrm{CH}_{4}$ separation. Journal of Materials Chemistry A, 2018, 6(16): $6847-6853$.

[101] LI S, FALCONER J L, NOBLE R D. Improved SAPO-34 membranes for $\mathrm{CO}_{2} / \mathrm{CH}_{4}$ separations. Advanced Materials, 2006, 18(19): 2601-2603.

[102] SHI H. Synthesis of SAPO-34 zeolite membranes with the aid of crystal growth inhibitors for $\mathrm{CO}_{2}-\mathrm{CH}_{4}$ separation. New Journal of Chemistry, 2014, 38(11): 5276-5278.

[103] SHI H. Organic template-free synthesis of SAPO-34 molecular sieve membranes for $\mathrm{CO}_{2}-\mathrm{CH}_{4}$ separation. RSC Advances, 2015, 5(48): 38330-38333.

[104] LI M, ZHANG J, LIU X, et al. Synthesis of high performance SAPO-34 zeolite membrane by a novel two-step hydrothermal synthesis+dry gel conversion method. Microporous and Mesoporous Materials, 2016, 225: 261-271.

[105] BAI L, CHANG N, LI M, et al. Ultrafast synthesis of thin SAPO-34 zeolite membrane by oil-bath heating. Microporous and Mesoporous Materials, 2017, 241: 392-399.

[106] KOSINOV N, GASCON J, KAPTEIJN F, et al. Recent developments in zeolite membranes for gas separation. Journal of Membrane Science, 2016, 499: 65-79. 